\title{
Impacts of satellite galaxies on the redshift-space distortions
}

\author{
Chiaki Hikage $^{1}$ and Kazuhiro Yamamoto ${ }^{2,3}$ \\ ${ }^{1}$ Kobayashi-Maskawa Institute, Nagoya University, Nagoya 464-8602, Japan \\ ${ }^{2}$ Department of Physical Sciences, Hiroshima University, Higashi-hiroshima, \\ Kagamiyama 1-3-1, 739-8526, Japan \\ ${ }^{3}$ Hiroshima Astrophysical Science Center, Hiroshima University, Higashi-Hiroshima, \\ Kagamiyama 1-3-1, 739-8526, Japan \\ E-mail: hikage@kmi.nagoya-u.ac.jp, kazuhiro@hiroshima-u.ac.jp
}

\begin{abstract}
We study the impacts of the satellite galaxies on the redshift-space distortions. In our multipole power spectrum analysis of the luminous red galaxies (LRGs) samples of the Sloan digital sky survey (SDSS), we have clearly detected the non-zero signature of the hexadecapole and tetrahexadecapole spectrum, which almost disappears in the power spectrum with the sample of the brightest LRGs only. We thus demonstrate that the satellite LRGs in multiple systems make a significant contribution to the multipole power spectrum though its fraction is small. The behavior can be understood by a simple halo model, in which the one-halo term, describing the Finger of God (FoG) effect from the satellite galaxies, makes the dominant contribution to the higher multipole spectra. We demonstrate that the smallscale information of higher multipole spectrum is useful for calibrating the satellite FoG effect and improves the measurement of the cosmic growth rate dramatically. We further demonstrate that the fiber collision in the galaxy survey influences the one-halo term and the higher multipole spectra, because the number of satellite galaxies in the halo occupation distribution (HOD) is changed. We also discuss about the impact of satellite galaxies on future high-redshift surveys targeting the $\mathrm{H}$-alpha emitters.
\end{abstract}

Keywords: power spectrum, redshift surveys, cosmic flows, modified gravity

ArXiv ePrint: 1303.3380 


\section{Contents}

1 Introduction 1

2 Impacts of satellite galaxies on the redshift-space distortions 3

2.1 Multipole power spectrum 4

2.2 Impact on parameter estimation $\quad 6$

3 Halo model description of satellite Finger-of-God $\quad 7$

4 Constraints on the growth rate and the properties of satellite galaxies $\quad 15$



5.1 HOD of $\mathrm{H} \alpha$ emitters 19

$\begin{array}{lll}5.2 & \text { Fisher matrix } & 20\end{array}$

6 Summary and Conclusions $\quad 23$

$\begin{array}{lr}\text { A Derivation of power spectrum } & 24\end{array}$

\section{Introduction}

The luminous red galaxies (LRGs) in the Sloan digital sky survey (SDSS) demonstrated the usefulness of a large redshift-survey of galaxies. Especially, it proved that a precise measurement of the statistical features in their spatial distribution provides us with the very useful methodology not only for the cosmology but also for the fundamental physics. For example, the baryon acoustic oscillation signature in the large scale structure is now recognized as a promising way for exploring the origin of the accelerated expansion of the universe $[1,2]$. A stringent constraint on the neutrino mass is also obtained [3, 4]. Furthermore, the LRG sample showed that a measurement of the redshift-space distortions gives us a unique chance of testing the theory of gravity (e.g., $[5,6]$, cf. [7]).

The LRGs in SDSS are massive early-type galaxies, and most part of them are considered to be residing in the center of halos. However, it is clarified that the some fraction of the LRGs consists of multiple galaxies system. The halo occupation distribution (HOD) of the LRGs was clarified by Reid and Spergel ([8], cf. [9]). The correspondence between the LRGs and halos has been investigated and has illuminated the importance of the Finger of God (FoG) effect [3, 10-12], which is non-linear redshift distortion due to the internal motion of galaxies within halos [13]. Recent investigations with N-body simulations have discovered that halos at the redshift 2 could be the origin of the LRG host halos [14]. In the present paper, we investigate the contribution of the satellite LRGs in multiple systems to the redshift-space distortions. A related topic has been investigated in the literature [3, 10-12], but the previous works investigated the contribution to the monopole spectrum. We here focus our investigation on the redshift-space distortions described by the higher multipole power spectrum.

The redshift-space distortions are measured in terms of the anisotropic correlation function or the anisotropic power spectrum, e.g., $[7,15,16]$. The anisotropic correlation function 
of the SDSS LRG sample has been measured in the literature, e.g., $[2,17,18]$. The anisotropic power spectrum $P(k, \mu)$, where $\mu$ denotes the directional cosine between the line of sight direction and the wave number vector, is the Fourier transform of the anisotropic correlation function. They are equivalent to each other. The multipole power spectrum $P_{\ell}(k)$ is defined as the coefficient of the multipole expansion, ${ }^{12}$

$$
P(k, \mu)=\sum_{\ell} P_{\ell}(k) \mathcal{L}_{\ell}(\mu)(2 \ell+1),
$$

or

$$
P_{\ell}(k)=\frac{1}{2} \int_{-1}^{+1} P(k, \mu) \mathcal{L}_{\ell}(\mu) d \mu
$$

where $\mathcal{L}_{\ell}(\mu)$ is the Legendre polynomial, which is normalized as

$$
\int_{-1}^{+1} \mathcal{L}_{\ell}(\mu) \mathcal{L}_{\ell^{\prime}}(\mu) d \mu=\frac{2}{2 \ell+1} \delta_{\ell \ell^{\prime}}
$$

In refs. [5, 21], the multipole power spectrum of the SDSS LRG sample was measured. In the present paper, we demonstrate that the satellite galaxies make a significant contribution to the higher multipole power spectrum, though its fraction is small.

The primary purpose of the present paper is to understand the contribution of satellite galaxies to the multipole power spectrum. To this end, we measure the multipole power spectrum of the SDSS LRG samples, and compare the results with the predictions of a simple halo model with the HOD of the SDSS LRG catalog. Then, we show the importance of the one halo term in the higher multipole power spectrum. We demonstrate that the information of multipole power spectra such as hexadecapole $P_{4}(k)$ and tetrahexadecapole $P_{6}(k)$ are useful for calibrating the satellite properties and significantly improve the measurement of the growth rate. We also investigate the influence of satellite galaxies in a future redshift survey targeting $\mathrm{H}$-alpha emitters on the multipole power spectrum in a measurement of the redshift-space distortions, because their contamination could give rise to a systematic error when comparing with theoretical models. An assessment of the systematic error is also the purpose of the present paper.

This paper is organized as follows. In section 2, we show the multipole power spectrum of the satellite LRGs and their contribution to the total LRG sample and the impact on the growth rate measurement. In section 3, we introduce a simple halo model for a system consisting of central galaxies and satellite galaxies, then we show that the halo model with the HOD of LRGs explains the behavior of the LRG multipole spectra. 4, we demonstrate a constraint from the LRG samples by comparing the observational results and the theoretical model. In section 5, we also discuss about the impact of satellite galaxies in a future survey targeting $\mathrm{H}$-alpha emitters at high redshifts. Section 6 is devoted to summary and conclusions. Appendix outlines the derivation of our theoretical expression for the multipole power spectrum in redshift space in the halo model. Throughout the present paper, we adopt the Hubble constant $H_{0}=100 h \mathrm{~km} / \mathrm{s} / \mathrm{Mpc}$ with $h=0.7$ unless otherwise stated.

\footnotetext{
${ }^{1}$ Note that the definition of $P_{\ell}(k)$ is different from the conventional definition by the factor $2 \ell+1$.

${ }^{2}$ There are also different works using phase-space distribution function approach to study the redshift distortion effect on the multipole power spectrum $[19,20]$.
} 


\begin{tabular}{ccc}
\hline \hline Name of LRG sample & Total Number of LRGs & Number of non-brightest LRGs \\
\hline All & 96762 & 4716 \\
BLRG & 92046 & 0 \\
Single & 87889 & 0 \\
NBLRG & 0 & 4716 \\
\hline
\end{tabular}

Table 1. Properties of LRG samples: "All" include all of LRGs in the SDSS DR7 LRG Sample in Northern sky; "BLRG" includes the brightest LRG in each group and the fainter LRGs are excluded; "Single" includes LRGs in single systems only, and any LRGs in multiple LRG systems are excluded; "NBLRG" consists of LRGs in multiple LRG systems except for the brightest LRGs.

\section{Impacts of satellite galaxies on the redshift-space distortions}

In this section, we demonstrate the contribution of the satellite LRGs to the multipole power spectrum. We here use the halo sample described in [12] using observed SDSS DR7-Full LRG sample in Northern sky (publicly available catalog prepared by [22]). The sample consists of 96762 LRGs with the magnitude $-23.2<M_{g}<-21.2$ in the redshift range $0.16<z<0.47$ (the mean redshift is 0.32 ) covering $1.44(\mathrm{Gpc} / \mathrm{h})^{3}$ comoving volume. Halo is identified with the counts-in-cylinders techniques developed by [8]: two galaxies are considered neighbors when the transverse separation $\Delta r_{\perp} \leq 0.8 \mathrm{Mpc} / \mathrm{h}$ and the redshift difference $\Delta z /(1+z) \leq$ 0.006 corresponding to the velocity difference $\delta v_{p}=1800 \mathrm{~km} / \mathrm{s}$. The total number of halos is 92046. When the missing galaxies due to fiber collisions are taken into account, the total number of LRGs become 98991. If all of them are hosted by the same halos of the observed LRGs, the actual number of satellite LRGs becomes 6945 (7\%). Most of halos (95.5\%) occupy single LRG (hereafter we call them "single LRG systems") and the rest of them contain multiple LRGs ("multiple LRG systems"). The multiplicity distribution of LRGs in halos is listed in Table 1 of [12]. For the multiple LRG systems, we choose the



Figure 1. Histogram of the number density of the LRG samples. The black, blue, green and red curves show the number density of the All, BLRG, Single, and NBLRG, respectively. 



Figure 2. Multipole power spectra $P_{0}(k), P_{2}(k), P_{4}(k)$, and $P_{6}(k)$ for the All LRG sample (black curve) and for the NBLRG (red diamond with large error bars). The squares with the small error bars show the results with the sample in a previous paper for comparison.

brightest LRG (BLRG) in each group as the central LRG and the rest of them are the nonbrightest LRGs (NBLRGs), which we regard as satellite LRGs. Strictly speaking, BLRGs are not always central LRGs as suggested by several observations (e.g., [12]), and our satellite sample contains central LRGs to some extent. We have used different samples described in Table 1 to see the impact of satellite galaxies on the redshift-space power spectrum.

Figure 1 shows the histogram of the number density of the galaxy samples as a function of the redshift $z$.

\subsection{Multipole power spectrum}

We adopt the method to measure the multipole power spectrum developed in [23]. For simplicity, we adopt the weight factor $\psi=1$, and the parameter $\alpha=0.1$ for the random catalog (see [23] for details). The method doesn't take the window effect of the survey region 



Figure 3. Same as Figure 2 but comparing the results with the All LRG sample (black curve), the BLRG sample (blue curve), and the Single LRG sample (green curve). The red curve is obtained by summing each component of the BLRG sample, the NBLRG sample and the cross correlation component. The agreement between the red curve and the black curve shows a consistency of the computation.

into account, but it is demonstrated that the window effect in our method is negligible by comparing with other method incorporating it explicitly [21]. We perform the multipole power spectrum analysis for each sample, whose results are shown in Figure 2 and 3.

Figure 2 compares the multipole power spectrum of the All LRG sample (black curve) and that of the NBLRGs (red diamond with large error bars). The squares with small error bars are the results in a previous work in [6], which are obtained from the LRG sample with 7150 square degrees sky coverage with the total number 100157 in the range of redshift $0.16 \leq z \leq 0.47$. Thus the previous sample is almost same as the "All" LRG sample in the 

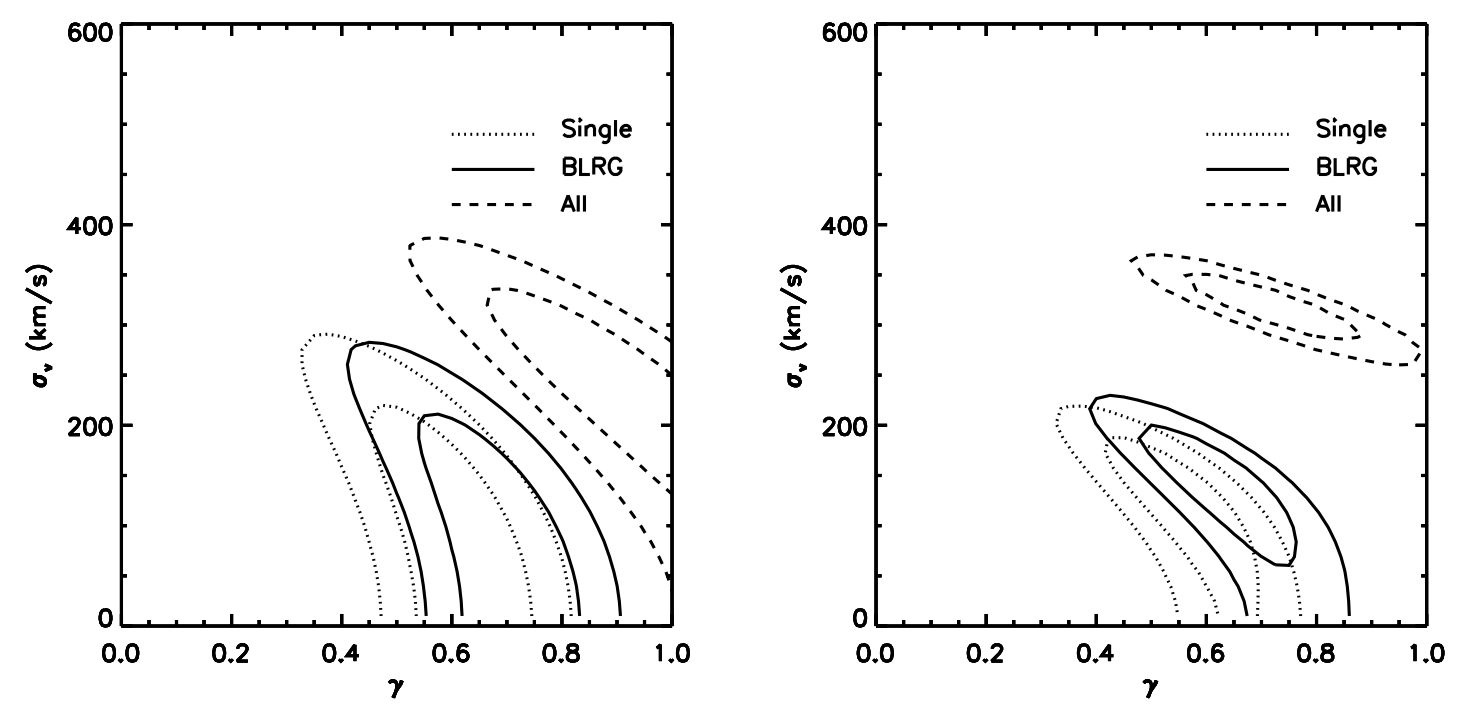

Figure 4. Contour of $\Delta \chi^{2}$ on $\widetilde{\sigma}_{v}$ and $\gamma$ plane. The solid (dotted) curves are the 1 sigma and the 2 sigma contours with the power spectrum with the brightest (single) LRG sample, while the dashed curve is the same but with the All LRG sample. The left panel used the data in the range of wavenumbers $0.01 h \mathrm{Mpc}^{-1} \leq k \leq 0.2 \mathrm{hpcc}^{-1}$, but the right panel used the data in the range $0.01 h \mathrm{Mpc}^{-1} \leq k \leq 0.3 h \mathrm{Mpc}^{-1}$.

present paper. This figure shows that the amplitude of the correlation of the NBLRGs is quite large compared with the dominant component.

Figure 3 shows the effect of the contamination of the NBLRG on the multipole power spectrum. The black curve is the results with the All LRG sample, the green curve is the one with the Single LRG sample, and the blue curve is the one with the BLRG sample. The difference between the green and blue is small, which means that the difference between the Single LRG sample and the BLRG sample is small. But the difference between the black curve and the blue curve is significant, which means that the contribution from the NBLRG sample is crucial though the fraction of the NBLRGs are small. This feature is significant for $P_{2}(k)$ and $P_{4}(k)$, especially. Thus, the contamination of the satellite galaxy is quite important in these multipole power spectra.

\subsection{Impact on parameter estimation}

Here let us demonstrate the impact of the contamination from the satellite galaxies (NBLRGs) in an estimation of cosmological parameters. For simplicity, let us consider the simple model of the anisotropic power spectrum

$$
P(k, \mu)=\left(b(k)+f \mu^{2}\right)^{2} P_{\mathrm{m}}^{\mathrm{NL}}(k) \mathcal{D}\left[k \mu \widetilde{\sigma}_{v} / H_{0}\right],
$$

where $P_{\mathrm{m}}^{\mathrm{NL}}(k)$ denotes a nonlinear matter power spectrum, $\mathcal{D}\left[k \mu \widetilde{\sigma}_{v} / H_{0}\right]$ is the damping factor due to the FoG effect and $\widetilde{\sigma}_{v}^{2}$ is the velocity dispersion parameter, for which we adopt the function

$$
\mathcal{D}[x]=\frac{1}{1+x^{2} / 2} .
$$


Here we determined the bias $b(k)$ so that the observational and the theoretical monopole spectra match. Then computed the chi-squared using the quadrupole spectrum by $\chi^{2}=$ $\sum_{i}\left[P_{2}^{\text {obs. }}\left(k_{i}\right)-P_{2}^{\text {theo. }}\left(k_{i}\right)\right]^{2} /\left[\Delta P_{2}^{\text {obs. }}\left(k_{i}\right)\right]^{2}$, where $P_{2}^{\text {obs. }}\left(k_{i}\right)$ and $\Delta P_{2}^{\text {obs. }}\left(k_{i}\right)$, are the observed values and errors, and $P_{2}^{\text {theo. }}\left(k_{i}\right)$ is the corresponding theoretical value. See reference [5] for details.

Figure 4 shows the 1 sigma and 2 sigma contours of $\Delta \chi^{2}$ on the parameter plane $\widetilde{\sigma}_{v}$ and $\gamma$, where the growth factor and the growth rate are parametrized as

$$
\begin{aligned}
& D_{1}(a)=a \exp \left[\int_{0}^{a} \frac{d a^{\prime}}{a^{\prime}}\left(\Omega_{m}\left(a^{\prime}\right)^{\gamma}-1\right)\right], \\
& f(a)=\frac{d \log D_{1}(a)}{d \log a}=\Omega_{m}(a)^{\gamma},
\end{aligned}
$$

where $\Omega_{m}(a)$ is the matter density parameter at the scale factor $a$. Here we fixed the other parameters $n_{s}=0.97, \Omega_{m}=0.28, \Omega_{b}=0.046, \sigma_{8}=0.8$ and assumed the cold dark matter model with a cosmological constant $(\Lambda \mathrm{CDM}$ model) as the background universe model. In each panel, the dotted curve, solid curve, and the dashed curve are the Single, Brightest, and All LRG sample, respectively. The left (right) panel used the data with $k \leq 0.2 \mathrm{hppc}^{-1}$ $\left(k \leq 0.3 h \mathrm{Mpc}^{-1}\right)$. The value $\gamma=0.55$ is the prediction of the model on the basis of the general relativity [24]. Though our theoretical model is very simple, the results clearly show that the contamination of the satellite galaxies (NBLRGs) significantly biases the parameter estimation. This figure also indicates that the results are influenced by including the brightest LRGs consisting of the multiple systems.

\section{Halo model description of satellite Finger-of-God}

In this section, we consider the FoG effect of satellite galaxies based on the halo model picture [25-27]. In the halo model, the power spectrum of LRGs are decomposed into 1-halo and 2 -halo terms. Then we write the anisotropic power spectrum in the redshift-space consisting of the 1-halo and 2-halo terms,

$$
P_{\mathrm{LRG}}(k, \mu)=P^{1 \mathrm{~h}}(k, \mu)+P^{2 \mathrm{~h}}(k, \mu) .
$$

We here consider the sample which consists of the central galaxies and the satellite galaxies, and adopt the following expressions (3.2) and (3.11) for $P^{1 \mathrm{~h}}(k, \mu)$ and $P^{2 \mathrm{~h}}(k, \mu)$, respectively. A brief summary of the derivation for a general case is described in the appendix (See also below for details).

One-halo term is given by

$$
P^{1 \mathrm{~h}}(k, \mu)=\frac{1}{\bar{n}^{2}} \int d M \frac{d n}{d M}\left[2\left\langle N_{\text {cen }}\right\rangle\left\langle N_{\text {sat }}\right\rangle \tilde{p}_{\mathrm{cs}}(k, \mu ; M)+\left\langle N_{\text {sat }}\left(N_{\text {sat }}-1\right)\right\rangle \tilde{p}_{\mathrm{ss}}(k, \mu ; M)\right],
$$

where we adopt the halo mass function $d n / d M$ given by [28] and $\bar{n}$ is the mean number density of LRGs given by $\bar{n}=\int d M(d n / d M) N_{\mathrm{HOD}}(M)$ and $N_{\mathrm{HOD}}(M)$ is the halo occupation distribution (i.e., the average number of galaxies inside the halo with mass $M$ ). We use the following form of the HOD of central LRGs and satellite LRGs [29]

$$
\begin{aligned}
& N_{\mathrm{HOD}}(M)=\left\langle N_{\mathrm{cen}}\right\rangle\left(1+\left\langle N_{\mathrm{sat}}\right\rangle\right), \\
& \left\langle N_{\mathrm{cen}}\right\rangle=\frac{1}{2}\left[1+\operatorname{erf}\left(\frac{\log _{10}(M)-\log _{10}\left(M_{\mathrm{min}}\right)}{\sigma_{\log M}}\right)\right], \\
& \left\langle N_{\mathrm{sat}}\right\rangle=f_{\mathrm{col}}(M)\left(\frac{M-M_{\mathrm{cut}}}{M_{1}}\right)^{\alpha},
\end{aligned}
$$




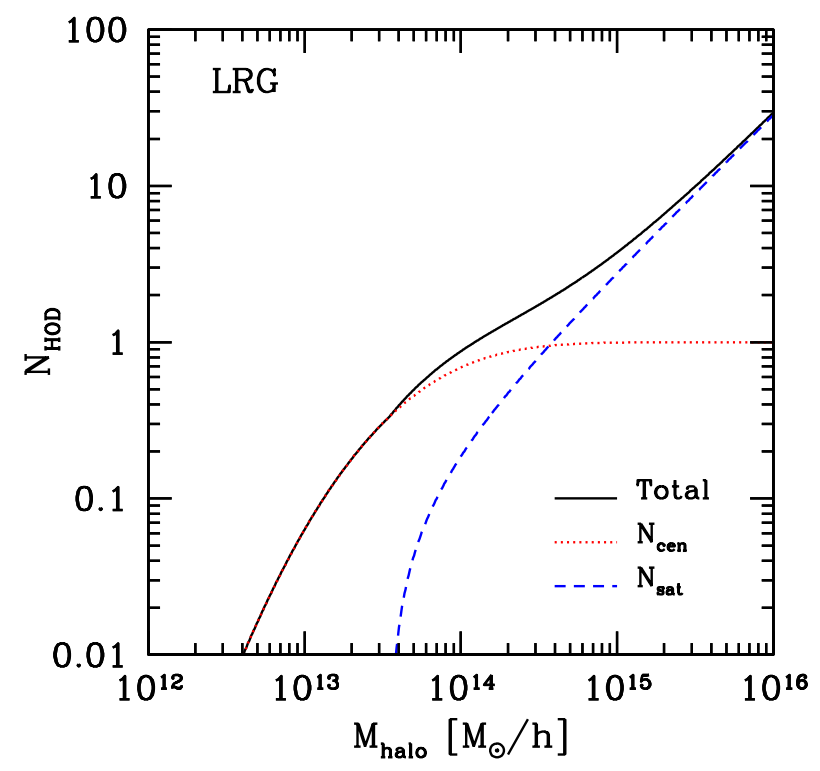

Figure 5. HOD for LRGs based on [8].

where $\operatorname{erf}(x)$ is the error function. We adopt $M_{\min }=5.7 \times 10^{13} M_{\odot} / h, \sigma_{\log M}=0.7, M_{\text {cut }}=$ $3.5 \times 10^{13} M_{\odot} / h, M_{1}=3.5 \times 10^{14} M_{\odot} / h$, and $\alpha=1$ to match the HOD of SDSS DR7 LRG catalog [8] as shown in Figure 5. Assuming the number of groups with $N_{\text {sat }}$ satellites is Poisson distributed [30], the averaged satellite-satellite pair number $\left\langle N_{\text {sat }}\left(N_{\text {sat }}-1\right)\right\rangle$ per halo goes to $\left\langle N_{\text {cen }}\right\rangle\left\langle N_{\text {sat }}\right\rangle^{2}$. We also take into account the missing galaxies due to the fiber collision by multiplying the satellite HOD with a following mass-dependent factor

$$
f_{\mathrm{col}}(M)=A_{\mathrm{col}}+B_{\mathrm{col}}\left(\frac{M-M_{\mathrm{cut}}}{M_{1}}\right)
$$

where $1-f_{\text {col }}(M)$ represent the fraction of missing satellite LRGs due to the fiber collision effect for the host halo mass of $M$. The factor $A_{\text {col }}$ and $A_{\text {col }}+B_{\text {col }}$ corresponds to $f_{\text {col }}(M)$ for $M=M_{\text {cut }}$ and $M=M_{1}$ where the averaged number of satellites is 0 and 1 respectively. Here we set $A_{\text {col }}=0.7$ and $B_{\mathrm{col}}=-0.05$ to match the number fraction of NBLRGs and the number of NBLRG pairs in groups. We do not consider the fiber collision effect on central HOD, for simplicity.

Central LRGs locate near the halo center and thus their velocity difference relative to the host halo should be small. Note that it is difficult to verify that each central LRG is located at the center of each halo in observational data. However, 20-40\% of brightest LRGs are found to be off-centered (satellite) galaxies using lensing and cross-correlation analysis [12]. Therefore, large part of the NBLRGs are off-centered and their velocity should be the main source of the FoG effect. The functions $\tilde{p}_{\mathrm{cs}}(k, \mu ; M)$ and $\tilde{p}_{\mathrm{ss}}(k, \mu ; M)$ are the Fourier transform of central-satellite and satellite-satellite distribution inside the halo with the mass of $M$, and the internal motion of satellite LRGs elongate the distributions in the line-ofsight direction. We assume that the internal velocity of the satellite LRGs has a Gaussian distribution determined by virial velocity as $\sigma_{v \text {,off }}(M)=\left(G M / 2 R_{\mathrm{vir}}\right)^{1 / 2}$, in which the virial radius of the halo with mass of $M$ is $R_{\mathrm{vir}}=\left(3 M / 4 \pi \bar{\rho}_{\mathrm{m}}(z) \Delta_{\mathrm{vir}}(z)\right)^{1 / 3}$ with $\Delta_{\mathrm{vir}}=265$ 




Figure 6. Pairwise velocity distribution between central-satellite (left) and satellite-satellite (right). Histogram indicates the observed pairwise velocity distribution obtained from the redshift differences between BLRGs and NBLRGs (left) and among NBLRGs (right) in the same groups. The value of $\sigma_{\Delta v}$ represent the r.m.s of the averaged pairwise velocity dispersion. For comparison, we plot the theoretical predictions based on the halo model (solid red curves), equation (3.9) in the left panel and equation (3.10) in the right panel, and an exponential profile with the dispersion of observed value of $\sigma_{\Delta v}$ (blue dashed curves).

at $z=0.32$. When the satellite motion is uncorrelated with each other, $\tilde{p}_{\mathrm{cS}}(k, \mu, M)$ and $\tilde{p}_{\mathrm{ss}}(k, \mu, M)$ are given by

$$
\begin{aligned}
& \tilde{p}_{\mathrm{cS}}(k, \mu, M)=\tilde{u}_{\mathrm{NFW}}(k ; M) \exp \left[-\frac{\sigma_{v, \mathrm{off}}^{2}(M) k^{2} \mu^{2}}{2 a^{2} H^{2}(z)}\right], \\
& \tilde{p}_{\mathrm{sS}}(k, \mu, M)=\tilde{p}_{\mathrm{cs}}^{2}(k, \mu, M) .
\end{aligned}
$$

We assume that the distribution of the satellite galaxies follows the NFW profile [31] and $\tilde{u}_{\mathrm{NFW}}(k)$ denotes the Fourier transform of truncated NFW profile, equation (A.4), (see also [32]). In order to test the validity of Gaussian assumption of satellite velocity distribution, equations (3.7) and (3.8), we compare the distribution functions of pairwise velocity for central-satellite pairs and satellite-satellite pairs based on the halo model, as shown in Figure 6. We compute the pairwise velocity between NBLRGs and BLRGs within the same group from their redshift difference as $\Delta v=c \Delta z /(1+z)$. We find that the distributions are well explained by the mass integral of the Gaussian velocity distribution with the Virial velocity 
dispersion of each mass $\sigma_{v \text {,off }}(M)=\left(G M / 2 R_{\text {vir }}\right)^{1 / 2}$,

$$
\begin{aligned}
& P(\Delta v)^{\text {cen-sat }} \propto \int d M \frac{d n}{d M}\left\langle N_{\text {cen }}\right\rangle\left\langle N_{\text {sat }}\right\rangle \exp \left(-\frac{\Delta v^{2}}{2 \sigma_{v, \text { off }}^{2}(M)}\right), \\
& P(\Delta v)^{\text {sat-sat }} \propto \int d M \frac{d n}{d M}\left\langle N_{\text {sat }}\left(N_{\text {sat }}-1\right)\right\rangle \exp \left(-\frac{\Delta v^{2}}{4 \sigma_{v, \text { off }}^{2}(M)}\right) .
\end{aligned}
$$

where the normalization of $P(\Delta v)$ is determined so that the integral over $\Delta v$ is unity. With the velocity probability distribution functions, we compute $\sigma_{\Delta v}^{2}=\int \Delta v^{2} P(\Delta v) d \Delta v$ for the theoretical value of pairwise velocity dispersions. The model predictions become $663 \mathrm{~km} / \mathrm{s}$ for central-satellite pairs and $937 \mathrm{~km} / \mathrm{s}$ for satellite-satellite pairs. These values well agree with the observed pairwise velocity dispersions: $653 \mathrm{~km} / \mathrm{s}$ for BLRG-NBLRG pairs and $909 \mathrm{~km} / \mathrm{s}$ for NBLRG-NBLRG pairs. The good agreement validates our models of central-satellite and satellite-satellite distributions in redshift space (equations (3.7) and (3.8)). For comparison, we also plot the exponential profile, which also well describes the behavior of the observed pairwise velocity distribution.

The 2-halo term is given by

$$
\begin{aligned}
P^{2 \mathrm{~h}}(k, \mu)= & {\left[\frac{1}{\bar{n}} \int d M \frac{d n}{d M}\left(b(M)+f \mu^{2}\right)\left\langle N_{\mathrm{cen}}\right\rangle\right.} \\
& \left.\times\left(1+\left\langle N_{\mathrm{sat}}\right\rangle \tilde{p}_{\mathrm{cs}}(k, \mu ; M)\right) \tilde{u}_{\mathrm{vol}}(k ; M)\right]^{2} P_{\mathrm{m}}^{\mathrm{NL}}(k),
\end{aligned}
$$

where $P_{\mathrm{m}}^{\mathrm{NL}}(k)$ is the real-space non-linear matter power spectrum. Here we use the non-linear matter power spectrum to describe the non-linear power spectrum of velocity divergence for simplicity, while the matter and velocity power spectra are actually different (c.f., $[36,37])$. Here we add the volume exclusion effect of halos $\tilde{u}_{\mathrm{vol}}(k ; M)$ in addition to the satellite distribution in order to include that two different halos cannot approach each other closer than a halo size. We use a Gaussian form $\tilde{u}_{\mathrm{vol}}(k ; M)=\exp \left(-\left(a k R_{\mathrm{vir}}(M)\right)^{2} / 2\right)$ and we choose the width parameter $a=2$ to fit the observed power spectrum.

Again we consider only the velocity distribution of satellite LRGs. We simply use the linear Kaiser formula [33] given by the term of $\left(b(M)+f \mu^{2}\right)$ with the growth rate $f \equiv d \ln D / d \ln a$ and the linear halo bias $b(M)[27,32,34]$. Without the FoG effect, that is $\sigma_{v \text {,off }}=0$, we have $P^{2 \mathrm{~h}} \simeq\left(b_{\text {eff }}+f \mu^{2}\right)^{2} P_{\mathrm{NL}}$ and $P^{1 \mathrm{~h}} \simeq N_{1 \mathrm{~h}}$, where $b_{\text {eff }}$ is the effective bias of LRGs given by $b_{\text {eff }}=\int d M(d n / d M) b(M) N_{\mathrm{HOD}}(M) / \bar{n}$ and $N_{1 \mathrm{~h}}$ is defined by $N_{1 \mathrm{~h}}=$ $\int d M(d n / d M)\left[2\left\langle N_{\text {cen }}\right\rangle\left\langle N_{\text {sat }}\right\rangle+\left\langle N_{\text {sat }}\left(N_{\text {sat }}-1\right)\right\rangle\right] / \bar{n}^{2}$. In this case, $N_{\text {lh }}$ is a constant and we have $P_{\ell}^{1 h}(k)=0$ for $\ell \geq 2$.

Figure 7 compares the halo model predictions of multipole power spectra for All LRG sample with the observations. Our model qualitatively well explain the observations although we simply adopted the linear Kaiser redshift distortion and the linear halo bias. The halo model well explains the differences between ALL and BLRG samples as shown in the below of this section. The satellite FoG effect in 1-halo term becomes significantly important at larger $k$ and dominant in the multipole spectra for $\ell \geq 4$ even though the satellite fraction is only $5 \%$. The 1-halo term contribution causes a systematic bias in the measurement of the growth rate as shown in Figure 4 because the FoG effects from the 1 and 2-halo terms have different feature and the simple form of equation (2.1) is not enough to describe both of the 

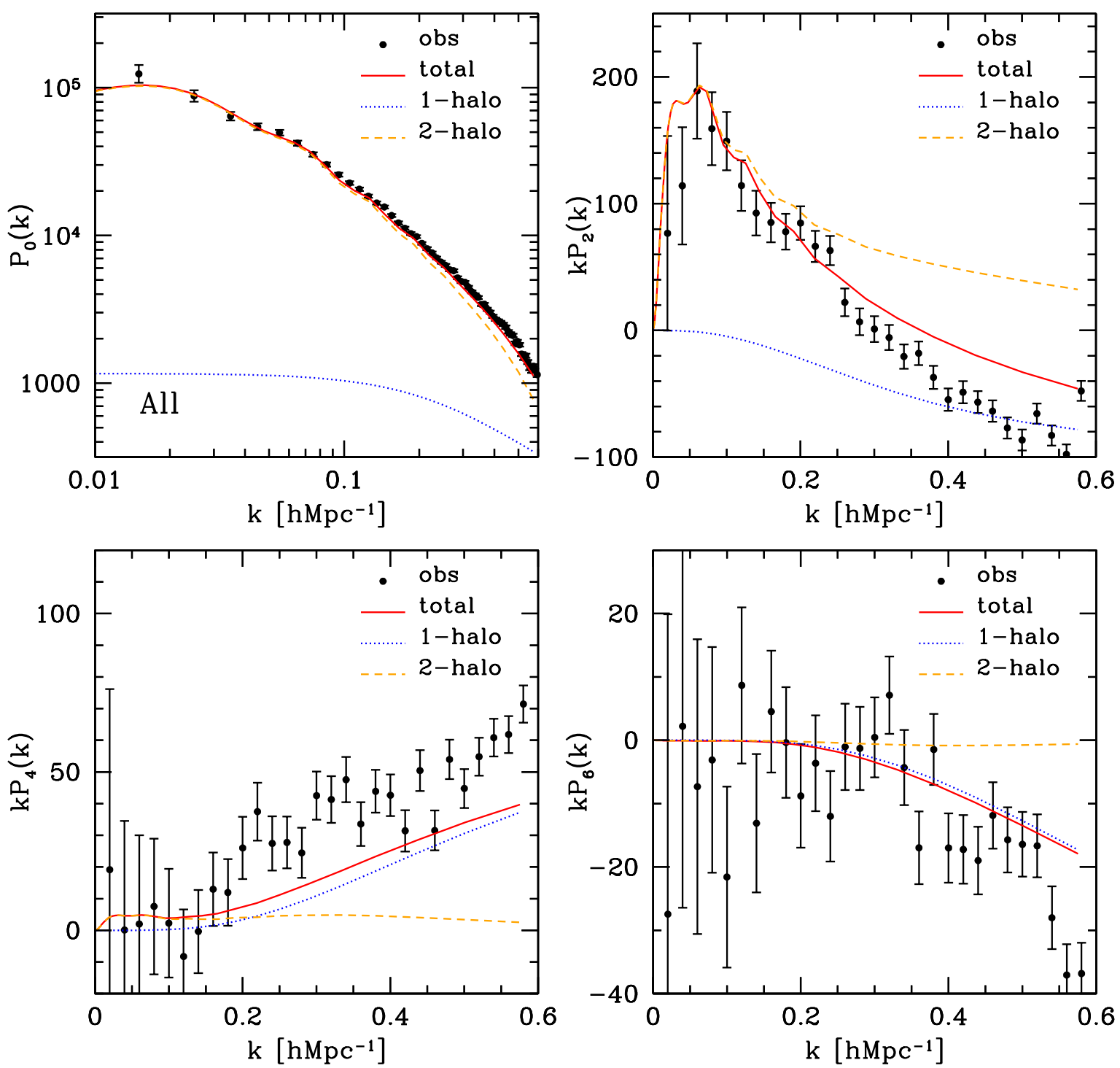

Figure 7. Halo model prediction for the multipole power spectra $P_{0}(k), P_{2}(k), P_{4}(k)$, and $P_{6}(k)$ for the All LRG sample. In each panel, the dotted curve and the dashed curve are the 1-halo term and the 2-halo term, respectively, and the solid curve is their combination. The black circles are the observational data of the All LRG sample in Figure 3.

FoG effects very well. In the following section, we show how the constraints on the growth rate changes by taking into account the 1-halo term.

The behavior of higher-order multipole spectrum is sensitive to the satellite FoG effect in one-halo term. In other words, the higher multipole spectra can be a good probe of the satellite fraction and the satellite velocity distribution. The one halo term making contribution to the multipole power spectrum of (3.2) can be written as follows:

$$
P_{\ell}^{1 h}(k)=\frac{1}{\bar{n}^{2}} \int d M \frac{d n}{d M}\left[2\left\langle N_{\mathrm{cen}}\right\rangle\left\langle N_{\mathrm{sat}}\right\rangle Q_{\ell}(q)+\left\langle N_{\mathrm{sat}}\left(N_{\mathrm{sat}}-1\right)\right\rangle Q_{\ell}(\sqrt{2} q)\right],
$$



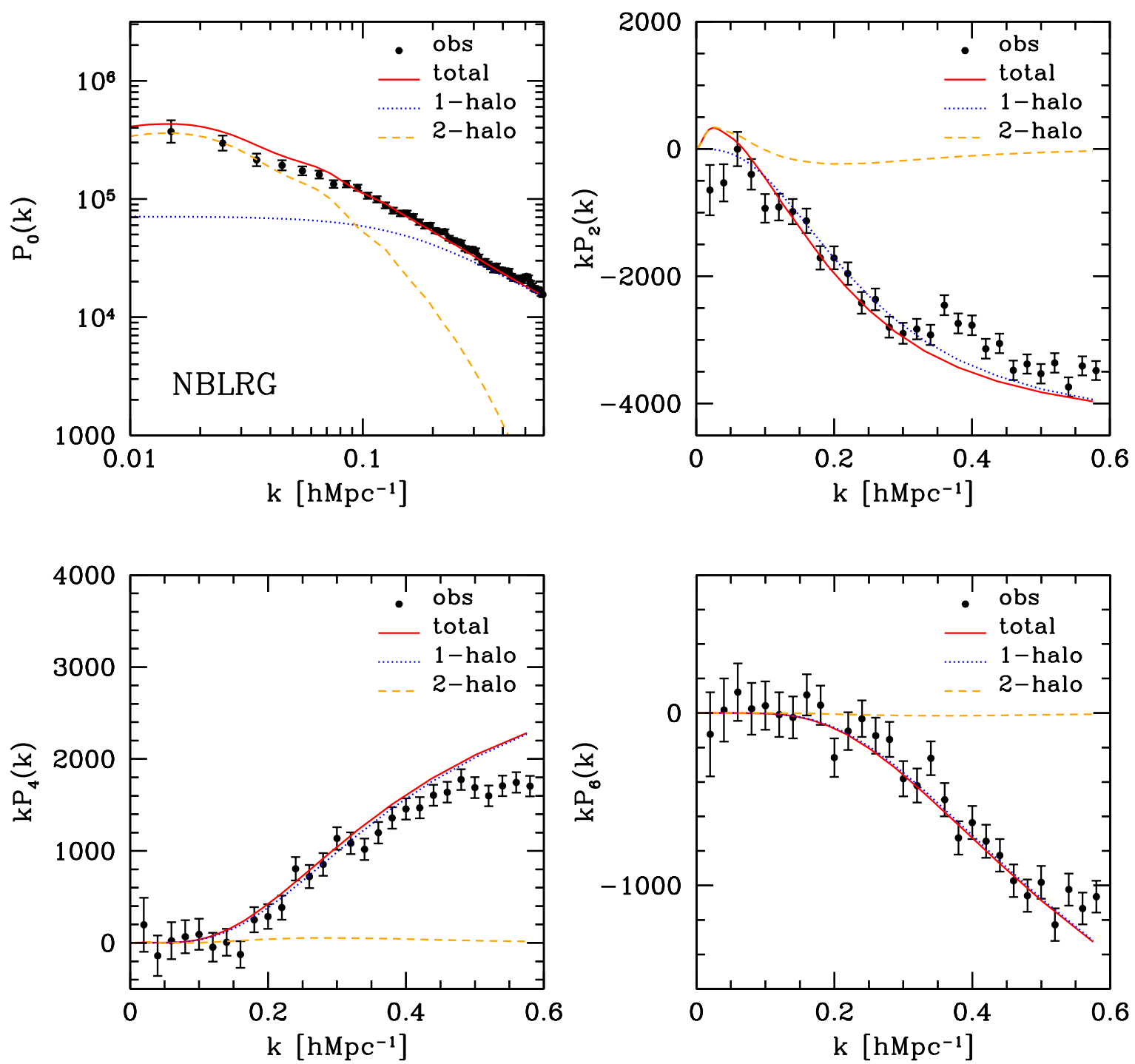

Figure 8. Halo model prediction for the multipole power spectra $P_{0}(k), P_{2}(k), P_{4}(k)$, and $P_{6}(k)$ for the NBLRG sample. In each panel, the dotted curve and the dashed curve are the 1-halo term and the 2-halo term, respectively, and the solid curve is their combination. Here the BLRG sample is assumed to be consisting of the central galaxies (35\%) and the satellite galaxies (65\%). The black circles are the observational data of the NBLRG sample in Figure 2.

where we defined

$$
Q_{\ell}(q)=\frac{1}{2} \int_{-1}^{1} d \mu e^{-q^{2} \mu^{2}} \mathcal{L}_{\ell}(\mu)
$$



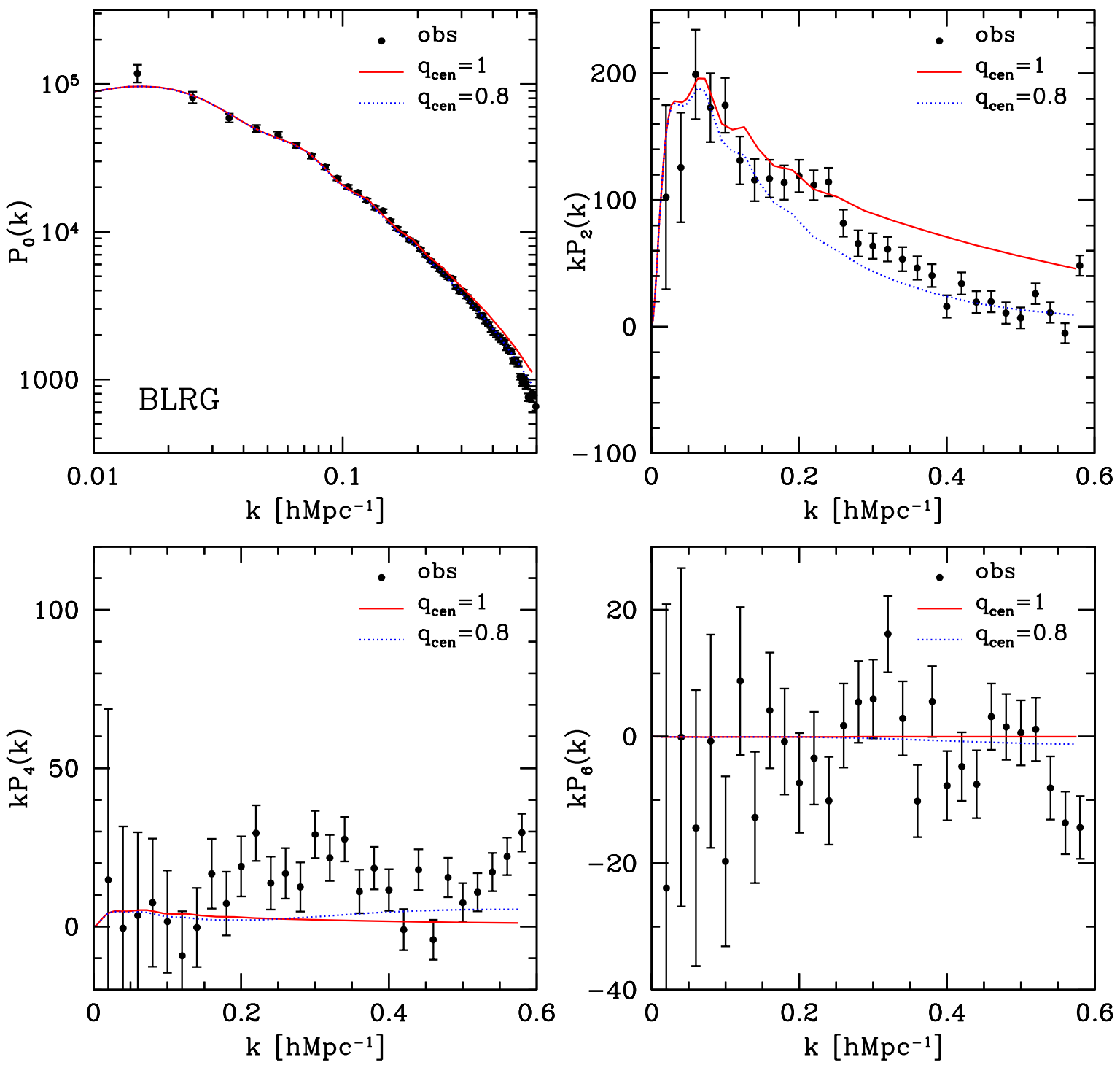

Figure 9. Halo model prediction for the multipole power spectra $P_{0}(k), P_{2}(k), P_{4}(k)$, and $P_{6}(k)$ for the BLRG sample, which is written with just 2-halo term. Here the solid curve adopted the fraction of the central galaxies $q_{\mathrm{cen}}^{(\mathrm{BLR})}=1$, while the dotted curve did $q_{\mathrm{cen}}^{(\mathrm{BLRG})}=0.8$.

and $q=\sigma_{v, \text { off }}(M) k / \sqrt{2} a H(z)$. Specifically, we have

$$
\begin{aligned}
& Q_{0}(q)=\frac{\sqrt{\pi}}{2 q} \operatorname{erf}(q), \\
& Q_{2}(q)=-\frac{3}{4 q^{2}} e^{-q^{2}}+\frac{\sqrt{\pi}\left(3-2 q^{2}\right)}{8 q^{3}} \operatorname{erf}(q), \\
& Q_{4}(q)=-\frac{5\left(21+2 q^{2}\right)}{32 q^{4}} e^{-q^{2}}+\frac{3 \sqrt{\pi}\left(35-20 q^{2}+4 q^{4}\right)}{64 q^{5}} \operatorname{erf}(q), \\
& Q_{6}(q)=-\frac{21\left(165+20 q^{2}+4 q^{4}\right)}{128 q^{6}} e^{-q^{2}}+\frac{5 \sqrt{\pi}\left(693-378 q^{2}+84 q^{4}-8 q^{6}\right)}{256 q^{7}} \operatorname{erf}(q) .
\end{aligned}
$$


The error function has the asymptotic form $\operatorname{erf}(q) \rightarrow 1$ for $q \gg 1$. Therefore, $Q_{\ell}(q)$ is in proportion to $(-1)^{\ell / 2} q^{-1}$ in the limit $q \gg 1$, which explains the asymptotic behavior of the multipole power spectrum at the large wave numbers. The central-satellite contribution is dominant in the 1-halo term of LRG samples and thus $k P_{\ell}^{1 \mathrm{~h}} \sim 2 k Q_{\ell}(q) f_{\text {sat }} / \bar{n}$ where $f_{\text {sat }}$ is the satellite fraction. When we use the values of $\bar{\sigma}_{v \text {,off }}=663 \mathrm{~km} / \mathrm{s}, a H(z)=88 \mathrm{hkm} / \mathrm{s}$ at $z=0.32, f_{\text {sat }}=0.07$ and $\bar{n} \simeq 10^{-4}(\mathrm{Mpc} / \mathrm{h})^{-3}$ for the LRG sample, the large-scale limit of $k P_{\ell}^{1 \mathrm{~h}}$ goes to 230 for $\ell=0,-120$ for $\ell=2,85$ for $\ell=4$, and -74 for $\ell=6$. Figure 7 shows that 1-halo term contribution approaches these values roughly.

Figure 8 compares the halo model predictions of the multipole power spectra for the "NBLRG" sample and the observed spectra. As $40 \%$ of BLRGs in multiple LRG systems are satellites (the number of multiple LRG systems $\mathcal{N}_{\text {mul }}$ is 4157), the same number of central galaxies are mixed in the NBLRG sample. Here we consider that $35 \%\left(=0.4 \mathcal{N}_{\text {mul }} / \mathcal{N}_{\text {sat }}\right)$ of the NBLRG sample are central galaxies and the rest of them are satellites. Based on this assumption we write the one-halo and two-halo terms of the power spectrum of the NBLRG sample as

$$
\begin{aligned}
& P^{\operatorname{NBLRG}}(k, \mu)=P^{1 \mathrm{~h}, \mathrm{NBLRG}}(k, \mu)+P^{2 \mathrm{~h}, \mathrm{NBLRG}}(k, \mu), \\
& P^{1 \mathrm{~h}, \mathrm{NBLRG}}(k, \mu)=\frac{1}{\bar{n}_{\mathrm{sat}}^{2}} \int d M \frac{d n}{d M}\left\langle N_{\mathrm{cen}}\right\rangle\left\langle N_{\mathrm{sat}}\right\rangle^{2} \\
& \times {\left[2 q_{\mathrm{cen}}^{(\mathrm{sat})}\left(1-q_{\mathrm{cen}}^{(\mathrm{sat})}\right) \tilde{p}_{\mathrm{cs}}(k, \mu ; M)+\left(1-q_{\mathrm{cen}}^{(\mathrm{sat})}\right)^{2} \tilde{p}_{\mathrm{ss}}(k, \mu ; M)\right], } \\
& P^{2 \mathrm{~h}, \mathrm{NBLRG}}(k, \mu)=\left[\frac{1}{\bar{n}_{\mathrm{sat}}} \int d M \frac{d n}{d M}\left(b(M)+f \mu^{2}\right)\left\langle N_{\mathrm{cen}}\right\rangle\left\langle N_{\mathrm{sat}}\right\rangle\right. \\
&\left.\times\left(q_{\mathrm{cen}}^{(\mathrm{sat})}+\left(1-q_{\mathrm{cen}}^{(\mathrm{sat})}\right) \tilde{p}_{\mathrm{cs}}(k, \mu ; M)\right) \tilde{u}_{\mathrm{vol}}(k ; M)\right]^{2} P_{\mathrm{m}}^{\mathrm{NL}}(k),
\end{aligned}
$$

where $q_{\mathrm{cen}}^{(\mathrm{sat})}$ is the fraction of central galaxies in the NBLRG sample. Here we set $q_{\mathrm{cen}}^{(\mathrm{sat})}=$ $\operatorname{Min}\left(0.35,1 /\left\langle N_{\text {sat }}\right\rangle\right)$ so that $q_{\text {cen }}^{\text {(sat) }}\left\langle N_{\text {sat }}\right\rangle$ does not exceed unity. The one-halo and two-halo terms for NBLRGs are plotted with the dotted curve and the dashed curve, respectively. The halo model explains the observed multipole spectral very well. One-halo term (dotted curve) is a dominant contribution to the multipole spectra and reaches $k P_{\ell}^{\mathrm{NBLRG}}(k) \sim \mathcal{O}\left(10^{3}\right)$.

The brightest LRG power spectrum does not have one-halo term because each halo contains one LRG at most. Then, the BLRG power spectrum is written only with the twohalo term. This can be clearly seen in that the BLRG multipole power spectra with $\ell=4$ and 6 are almost zero in Figure 9, which indicates that the 1-halo term from satellite galaxies becomes significantly smaller by removing NBLRGs. This means that the halo reconstruction method we use well succeeds in removing the one-halo term. This is important for the study of precision cosmology because the uncertainty of the satellite HOD and its FoG effect becomes significantly small. In a strict sense, however, $P_{4}(k)$ has slightly positive signature compared to the halo model predictions. This may come from that the reconstruction method is incomplete and some of satellite LRGs are included in the BLRG sample. Multipole spectra such as $P_{4}(k)$ dominated by the 1-halo term is useful for estimating the residual 1-halo term effect.

However, several observations indicate that some fraction of BLRGs are satellite or off-centered galaxies (e.g., [35]), which causes the FoG effect [11]. When the fraction that BLRGs locate on the mass center of their host halos is $q_{\mathrm{cen}}^{(\mathrm{BLRG})}$, the BLRG power spectrum 
is given

$$
\begin{aligned}
P^{\mathrm{BLRG}}(k, \mu)= & {\left[\frac{1}{\bar{n}} \int d M \frac{d n}{d M}\left(b(M)+f \mu^{2}\right)\left\langle N_{\mathrm{cen}}\right\rangle\right.} \\
& \left.\times\left(q_{\mathrm{cen}}^{(\mathrm{BLRG})}+\left(1-q_{\mathrm{cen}}^{(\mathrm{BLRG})}\right) \tilde{p}_{\mathrm{cs}}(k, \mu ; M)\right) \tilde{u}_{\mathrm{vol}}\right]^{2} P_{\mathrm{m}}^{\mathrm{NL}}(k) .
\end{aligned}
$$

As shown in [12], the lensing and cross-correlation measurements indicate $20 \%$ fraction of BLRGs are off-centered. Figure 9 shows the model predictions of the BLRG power spectra with $q_{\mathrm{cen}}^{(\mathrm{BLRG})}=1$ (all of BLRGs are centrals) and $q_{\mathrm{cen}}^{(\mathrm{BLRG})}=0.8(20 \%$ of BLRGs are satellite). The figure shows that their agreement is better at high $k$ when the satellite FoG effect is included, which indicates that even BLRG sample may have significant FoG effect on the multipole power spectra. Note that the result may change if we take into account the nonlinearity in the galaxy biasing. The linear Kaiser formula is the simplest model, then more careful analysis will be necessary using the sophisticated perturbation theories as well as numerical simulations ([36-39]), though such analysis is beyond the scope of the present paper.

Figure 10 shows the differences of multipole power spectra $P_{2}$ and $P_{4}$ between ALL and BLRG samples. The curves in each panel are the theoretical prediction of our model using the satellite HOD parameters of the NBLRG samples. The theoretical curves much better fit the observational results, compared with those in Figure 7. This agreement indicates the contamination of the FoG effect of the off-centered velocities in the BLRGs.

\section{Constraints on the growth rate and the properties of satellite galaxies}

In this section, we consider a constraint by comparing the observed multipole power spectrum of the LRG samples and our theoretical model including the one-halo term. This
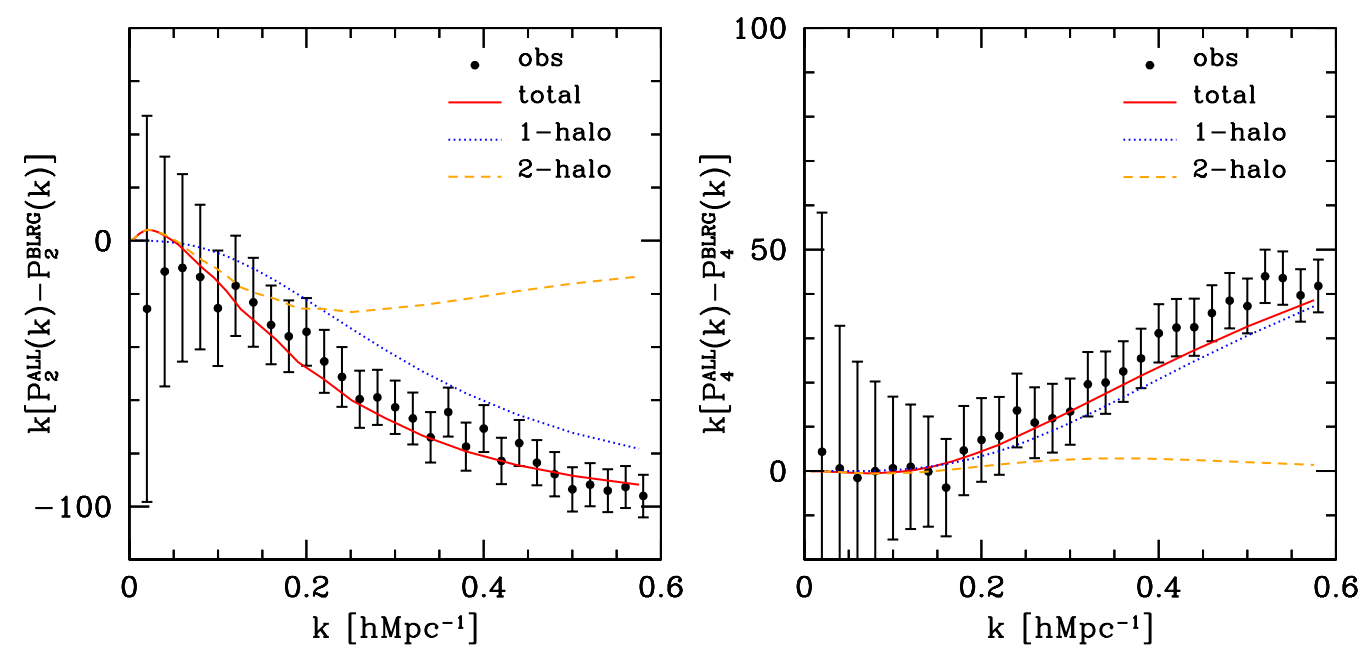

Figure 10. Differences of multipole power spectra $P_{2}$ (left) and $P_{4}$ (right) between All and BLRG samples. Sold curves show the halo model prediction, which mainly comes from the one-halo term (dotted curve) compared to the two-halo term (dashed curve). 
demonstrates how the one-halo term influences a cosmological constraint. We define $\chi^{2}$ by

$$
\chi^{2}=\sum_{\ell=0,2,4,6} \sum_{i} \frac{\left[P_{\ell}^{\text {obs. }}\left(k_{i}\right)-P_{\ell}^{\text {model }}\left(k_{i}\right)\right]^{2}}{\left[\Delta P_{\ell}\left(k_{i}\right)\right]^{2}},
$$

where $P_{\ell}^{\text {obs. }}\left(k_{i}\right)$ and $\Delta P_{\ell}\left(k_{i}\right)$ are the observed power spectrum and the error, respectively, and $P_{\ell}^{\text {model }}\left(k_{i}\right)$ is the theoretical model, described in the below.

Based on the halo model developed in previous section, we fit the observed power spectra with the following form of the power spectra averaged over halo mass

$$
\begin{aligned}
& P^{\text {model }}(k, \mu)=P^{1 \mathrm{~h}, \text { model }}(k, \mu)+P^{2 \mathrm{~h}, \text { model }}(k, \mu) \\
& P^{1 \mathrm{~h}, \text { model }}(k, \mu)=\frac{2 f_{\text {sat }}}{\bar{n}} \mathcal{D}\left(\frac{k \mu \bar{\sigma}_{v, \text { off }}}{a H}\right), \\
& P^{2 \mathrm{~h}, \text { model }}(k, \mu)=\left\{\left(\bar{b}(k)+f \mu^{2}\right)\left[\left(1-f_{\text {sat }}\right)+f_{\text {sat }} \mathcal{D}\left(\frac{k \mu \bar{\sigma}_{v, \text { off }}}{a H}\right)\right]\right\}^{2} P_{\mathrm{m}}^{\mathrm{NL}}(k),
\end{aligned}
$$

where $\bar{b}(k)$ is the averaged bias of LRGs and linearly fitted as $b_{0}+b_{1} k$. Here the growth rate is $f=\Omega_{m}(z)^{\gamma}$, assuming the $\Lambda$ CDM model as background universe, and the other cosmological parameters are fixed as $n_{s}=0.96, \Omega_{m}=0.28, \Omega_{b}=0.044, \sigma_{8}=0.8$. We consider the FoG of satellite LRGs and parametrize it with the satellite fraction $f_{\text {sat }}$ and the averaged velocity dispersion $\bar{\sigma}_{v \text {,off }}$. We use a Lorentzian form of FoG damping function of (2.2), which well approximates the observed satellite velocity distribution as shown in Figure 6 . In the limit of small $k$, the FoG damping function $\mathcal{D}(x)$, eq. (2.2), becomes $1-x^{2} / 2$. In this lowest-order approximation, $\widetilde{\sigma}_{v}^{2}$ in equation (2.1) corresponds to $2 f_{\text {sat }}\left(\bar{\sigma}_{v \text {, off }} H_{0} / a H(z)\right)^{2}$ in equation (4.4). For the 1-halo term, we only take the dominant contribution of the central-satellite pairs into account, and neglect that from the satellite-satellite pairs. We do not introduce additional parameter of central fraction (i.e., $q_{\mathrm{cen}}$ ), for simplicity, while it is still controversial issue whether BLRGs are off-centered or not. Instead, we leave $f_{\text {sat }}$ as a free parameter because the observed multipole power spectrum, $P_{4}(k)$ in Figure 9 , systematically deviates from zero even at small $k$, which suggests the residual 1-halo terms. In summary, the number of the fitting parameters is 5 in total: $b_{0}, b_{1}, \gamma, f_{\text {sat }}$, and $\bar{\sigma}_{v, \text { off }}$.

First, let's see how adding the 1-halo term in the theoretical model changes the fitting results. Table 2 compares the constraints on the parameters of $\gamma, f_{\text {sat }}, \bar{\sigma}_{v, \text { off }}$ without 1-halo term and those with 1-halo term for All, BLRG, and Single LRG samples, respectively. The fitting range is up to $k=0.2 \mathrm{~h} / \mathrm{Mpc}$ for all of $P_{l}(l=0,2,4,6)$, and the bias parameters are marginalized over. In the fitting (I) without 1-halo term, the value of $\gamma$ for All sample is overestimated (or growth rate $f$ is underestimated) compared to that of the BLRG or Single LRG sample, which is also shown in Figure 4: the difference of best-fit values of $\gamma$ between All and Single is 0.16 and that between BLRG and Single is 0.07. The deviation is mildly alleviated by including the 1-halo term in the fitting (II): 0.12 between ALL and Single and 0.04 between BLRG and Single. However, the 1-halo term effect is highly degenerated with the growth rate.

Next we add the information of the small-scale measurements of $P_{4}(k)$ and $P_{6}(k)$ of the range of wavenumbers up to $k=0.6 h / \mathrm{Mpc}$ in the fitting. As shown in the previous section, $P_{4}(k)$ and $P_{6}(k)$ at large $k$ is dominated by the FoG effect of 1-halo term, then the information can be used to calibrate the uncertainty of the satellite FoG. We find that the information of $P_{4}(k)$ and $P_{6}(k)$ on small scales (at large $k$ ) significantly improves the 


\begin{tabular}{cccccc}
\hline \hline & method & Sample & $\gamma$ & $100 f_{\text {sat }}$ & $\bar{\sigma}_{v, \text { off }}[\mathrm{km} / \mathrm{s}]$ \\
\hline \multirow{3}{*}{ (I) } & $k<0.2 h / \mathrm{Mpc}$ for all $P_{l}$ & All & $0.78 \pm 0.10$ & $20 \pm 21$ & $590 \pm 300$ \\
& w/o 1-halo term & BLRG & $0.69 \pm 0.07$ & $33 \pm 30$ & $210 \pm 250$ \\
& & Single & $0.62 \pm 0.06$ & $33 \pm 30$ & $200 \pm 220$ \\
\hline \multirow{3}{*}{ (II) } & $k<0.2 h / \mathrm{Mpc}$ for all $P_{l}$ & All & $0.72 \pm 0.10$ & $4.5 \pm 1.6$ & $910 \pm 180$ \\
& with 1-halo term & BLRG & $0.64 \pm 0.08$ & $1.2 \pm 1.3$ & $700 \pm 320$ \\
& & Single & $0.60 \pm 0.07$ & $1.0 \pm 1.5$ & $590 \pm 340$ \\
\hline \multirow{3}{*}{ (III) } & $k<0.2 h / \mathrm{Mpc}$ for $P_{0}, P_{2}$ & All & $0.54 \pm 0.04$ & $8.5 \pm 0.4$ & $780 \pm 50$ \\
& $k<0.6 h / M p c$ for $P_{4}, P_{6}$ & BLRG & $0.57 \pm 0.04$ & $2.4 \pm 0.4$ & $850 \pm 150$ \\
& with 1-halo term & Single & $0.56 \pm 0.04$ & $1.8 \pm 0.5$ & $830 \pm 190$ \\
\hline
\end{tabular}

Table 2. Constraints on the index of growth rate $\gamma$, satellite fraction $f_{\text {sat }}$ and averaged velocity dispersion $\bar{\sigma}_{v \text {,off }}$ from the fitting of the multipole power spectra $P_{l}(k)$ with $(l=0,2,4,6)$ for All, BLRG and Single LRG samples. In the fitting, we compare the three methods with and without 1halo term in the modeling and adopting the different range of wavenumbers: (I) fitting all $P_{l}(k)$ in the range of $k<0.2 h / \mathrm{Mpc}$ without 1-halo term (top); (II) fitting all $P_{l}(k)$ in the range of $k<0.2 h / \mathrm{Mpc}$ with 1-halo term (middle); (III) fitting $P_{0}(k)$ and $P_{2}(k)$ in the range of $k<0.2 h / \mathrm{Mpc}$ while $P_{4}(k)$ and $P_{6}(k)$ in the range of $k<0.6 h / \mathrm{Mpc}$ with 1 -halo term (bottom).

error of satellite fraction by a factor $3 \sim 4$ and the error of $\gamma$ by a factor 2 . Here $P_{4}(k)$ plays an important role, especially. Our constraints on $\gamma$ from the 3 different LRG samples becomes consistent with each other by including the higher multipole spectra at large $k$. This indicates that our fitting formula including 1-halo term well describes the behavior of three different LRG samples. Figure 11 shows the contour of the joint constraints on $\gamma$ and



Figure 11. Joint constraints on $\gamma$ and satellite fraction $f_{\text {sat }}$ from the fitting of the multipole power spectra $P_{l}(k)$ with $(l=0,2,4,6)$ for All, BLRG, and Single samples. In each panel, the (blue) large curves are the $1 \sigma$ and $2 \sigma$ contours with the data of the range $k<0.2 h / \mathrm{Mpc}$, while the (red) small circles are the same but with the data of the range $k<0.6 h / \mathrm{Mpc}$ for $P_{4}(k)$ and $P_{6}(k)$. The vertical dashed line shows $\gamma=0.55$, the prediction of the general relativity. 

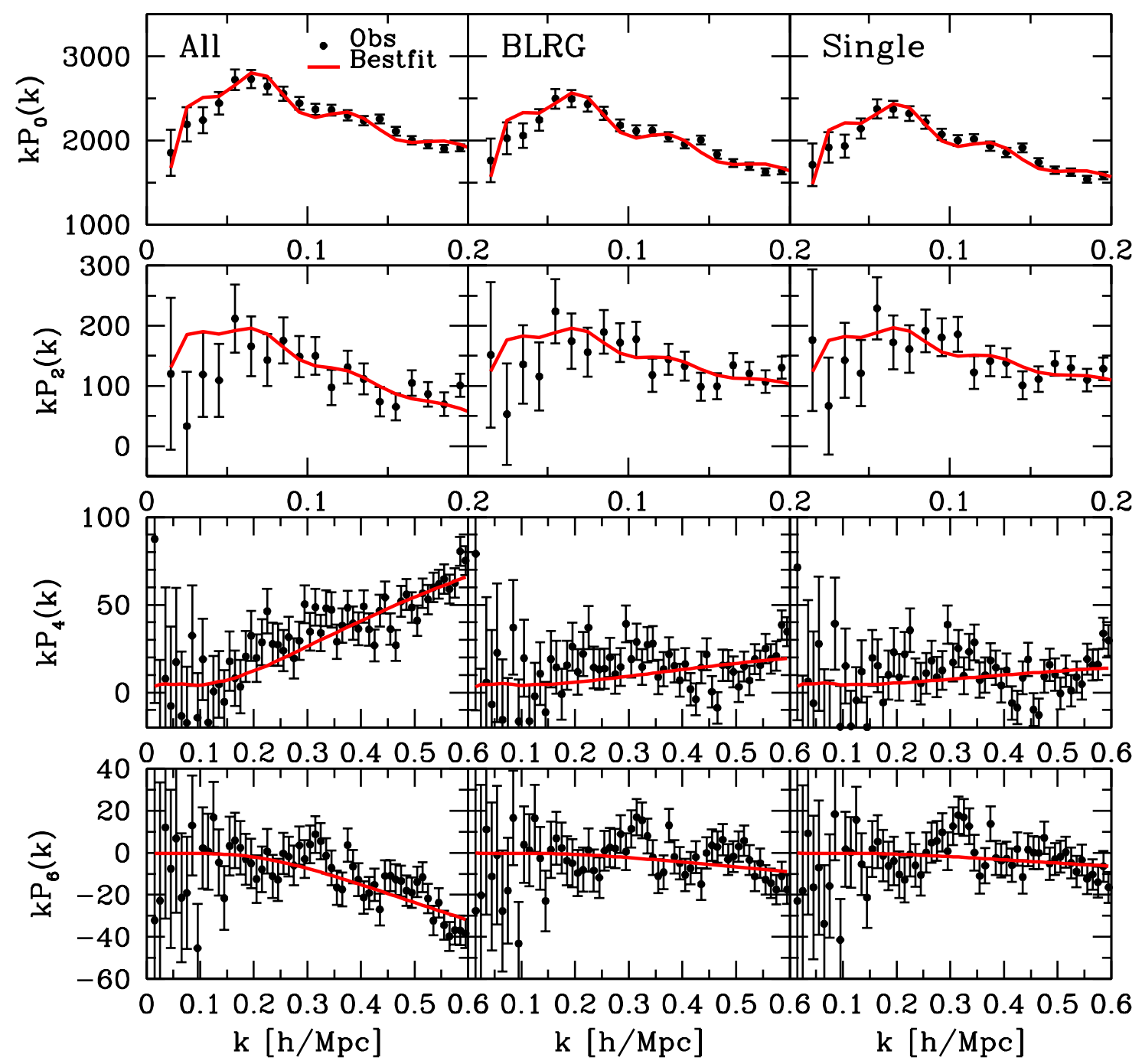

Figure 12. Comparison of $P_{0}(k), P_{2}(k), P_{4}(k)$ and $P_{6}(k)$ for All (left panel), BLRG (center panel), and Single (right panel) samples and the models with the best-fit parameters. The maximum value of $k$ is $0.2 h / \mathrm{Mpc}$ for $P_{0}(k)$ and $P_{2}(k)$, while $0.6 h / \mathrm{Mpc}$ for $P_{4}(k)$ and $P_{6}(k)$.

$f_{\text {sat }}$ when the small-scale information of $P_{4}(k)$ and $P_{6}(k)$ is included (red) and not included (blue). It is clearly seen that the measurements of $P_{4}(k)$ and $P_{6}(k)$ on small-scales break the degeneracy between $\gamma$ and $f_{\text {sat }}$ and improves their errors dramatically. Figure 12 compares the observations (black filled circles with error bars) of the multipole power spectra and the corresponding best-fitted curve (red solid curves). Our model well describes the observations of the three samples including $P_{4}(k)$ and $P_{6}(k)$ at large $k$. Actually the satellite fraction for BLRG and Single LRG samples significantly decreases, as described in Table 2. However, it still remains $\sim 2 \%$ fraction of central-satellite pair, accordingly the satellite fraction for All sample becomes $\sim 7 \%$, which is higher than the expected value including the fiber collision 
$\sim 5 \%$. This may indicate that the halo reconstruction is incomplete and some of satellite galaxies are still included in BLRG and Single LRG samples. Multipole power spectra such as $P_{4}(k)$ and $P_{6}(k)$ are a good indicator for the residual 1-halo term and may be useful for finding a better grouping method. Our constraint on the velocity dispersion of $\bar{\sigma}_{v \text {,off }}$ is $\sim 800 \mathrm{~km} / \mathrm{s}$, which is roughly consistent with the observed pairwise velocity dispersion between BLRG and NBLRGs, that is $653 \mathrm{~km} / \mathrm{s}$ as shown in Figure 6.

Our method using the measurements of higher multipole spectrum $P_{4}(k)$ provides a promising way to calibrate the satellite FoG effect and improve the error of the growth rate measurement. The measurements of the satellite fraction and the velocity dispersion can be translated to the constraints on the satellite HOD and/or the velocity bias between LRGs and halos. However, our theoretical model is still very simple and uses various approximations such as Kaiser approximation. In order to obtain more robust estimates on the growth rate and satellite properties, we need more precise theoretical models of halo clustering and velocity probability distribution function by comparing with simulated mock samples. Such detailed analysis is beyond the scope of this paper and is left as future work.

\section{Forecast on multipole power spectra for $\mathrm{H} \alpha$ emitters}

Main targets of high-redshift $(z=1 \sim 2)$ galaxy surveys, planed in such as Subaru/PFS [40] and Euclid [41], are $\mathrm{H} \alpha$ emitters (HAE). In this section, we perform Fisher analysis to estimate the impact of satellite galaxies for such future surveys targeting HAEs.

\section{$5.1 \quad$ HOD of $\mathbf{H} \alpha$ emitters}

The relation of HAEs to halos are less known observationally, and will be more complicated than that of LRGs. We use the following form of HOD based on the sample of 370 HAEs at $\mathrm{z}=2.23$ detected in Hi-Z Emission Line Surveys (HiZELs) [42]

$$
\begin{aligned}
\left\langle N_{\mathrm{cen}}^{H \alpha}\right\rangle & =F_{b}\left(1-F_{a}\right) \exp \left[-\frac{\left(\log _{10}(M)-\log _{10}\left(M_{\mathrm{min}, \mathrm{c}}\right)\right)^{2}}{2 \sigma_{\log M, \mathrm{c}}^{2}}\right] \\
& +F_{a}\left[1+\operatorname{erf}\left(\frac{\log _{10}(M)-\log _{10}\left(M_{\mathrm{min}, \mathrm{c}}\right)}{\sigma_{\log M, \mathrm{c}}}\right)\right], \\
\left\langle N_{\mathrm{sat}}^{H \alpha}\right\rangle & =f_{\mathrm{col}} F_{s}\left[1+\operatorname{erf}\left(\frac{\log _{10}(M)-\log _{10}\left(M_{\mathrm{min}, \mathrm{s}}\right)}{\sigma_{\log M, \mathrm{~s}}}\right)\right]\left(\frac{M}{M_{\text {min }, \mathrm{s}}}\right)^{\alpha} .
\end{aligned}
$$

Here the central HAE distribution is described with Gaussian and smoothed step-like components with their amplitudes determined by the normalization factors $F_{a}$ and $F_{b}$. The typical mass and the dispersion are parametrized with $M_{\min , \mathrm{c}}$ and $\sigma_{\log M, \mathrm{c}}$, respectively. Satellite HOD is described with a smoothed step-like component multiplied by power-law with scaling of $\alpha$, the typical satellite mass $M_{\min , \mathrm{s}}$ and the amplitude $F_{s}$. The values of HOD parameters for different luminosity samples are listed in Table. The plots of HODs are shown in Figure 13.

Figure 14 shows the comparison of multipole power spectra for central HAEs with those for all HAEs including satellites at $z=2.23$. The FoG effect on the HAE power spectrum from satellite in a halo is much smaller than that on LRGs power spectrum because the typical halo mass of HAEs is much smaller than that of LRGs. In our halo model, averaged virial velocity of halos hosting HAEs is $170 \mathrm{~km} / \mathrm{s}$, while those hosting LRGs are $660 \mathrm{~km} / \mathrm{s}$. For the faint HAE sample, the contamination of the satellite changes the higher multipole 




Figure 13. HOD for $\mathrm{H}_{\alpha}$ emitters based on [42]. Here we set no fiber collision effect $f_{\text {col }}=1$.

spectrum at a few percent or 10 percent level depending on the wave number, while the effect becomes smaller for the luminous HAE sample because the satellite fraction decreases. FoG effect for HAEs are expected to be much smaller than LRGs, however, upcoming galaxy surveys are expected to measure the growth rate measurement at the percent-level accuracy and thus it is still important to estimate the systematic errors of the FoG effect.

\subsection{Fisher matrix}

We here discuss about systematic errors from uncertainties of the satellite galaxies in future redshift survey at a quantitative level. To this end, we adopt the Fisher matrix technique to estimate the systematic errors from the one halo term (see, e.g., [11, 43, 44]). The bias in a parameter is estimated by

$$
\delta \theta_{i}=-\left[F^{\theta \theta}\right]_{i k}^{-1} F_{k j}^{\theta \psi} \delta \psi_{j},
$$

where $F_{i j}^{\theta \theta}$ is the Fisher matrix, whose inverse matrix is $\left[F^{\theta \theta}\right]_{i k}^{-1}$, and $F_{k j}^{\theta \psi} \delta \psi_{j}$ is a vector which describes the systematic bias caused by ignoring the one-halo term. In case $A$, we adopt the

Table 3. HOD parameters for HAEs

\begin{tabular}{lrrr}
\hline Luminosity & $L>10^{41} \mathrm{erg} / \mathrm{s}$ & $L>10^{42} \mathrm{erg} / \mathrm{s}$ & $L>10^{43} \mathrm{erg} / \mathrm{s}$ \\
\hline$F_{a}$ & 0.4 & 0.05 & 0.0035 \\
$F_{b}$ & 0.33 & 0.35 & 0.06 \\
$F_{s}$ & 0.1 & 0.02 & 0.001 \\
$M_{\min , \mathrm{c}}\left(10^{12} M_{\odot} / h\right)$ & 0.12 & 0.8 & 1 \\
$\sigma_{\log M, \mathrm{c}}$ & 0.14 & 0.25 & 0.22 \\
$M_{\min , \mathrm{s}}\left(10^{12} M_{\odot} / h\right)$ & 0.5 & 1.1 & 1 \\
$\sigma_{\log M, \mathrm{~s}}$ & 0.24 & 0.32 & 0.24 \\
$\alpha$ & 1 & 0.8 & 0.8 \\
\hline
\end{tabular}



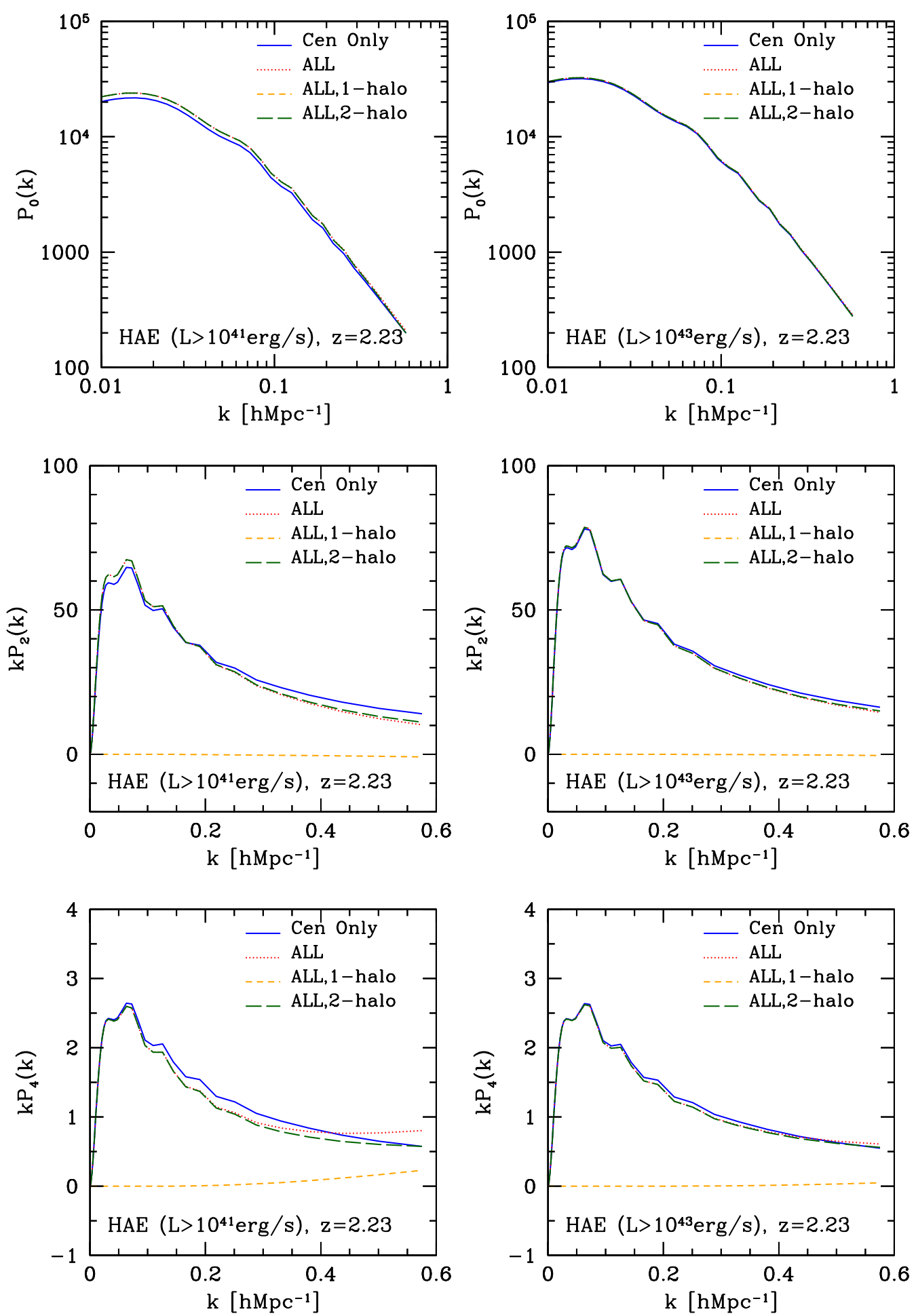

Figure 14. Multipole power spectra $P_{0}$ (top), $P_{2}$ (middle), and $P_{4}$ (bottom) for $\mathrm{H}_{\alpha}$ emitters with $L>10^{43} \mathrm{erg} / \mathrm{s}$ (left panels) and $L>10^{41} \mathrm{erg} / \mathrm{s}$ (right panels) at $z=2.23$. 

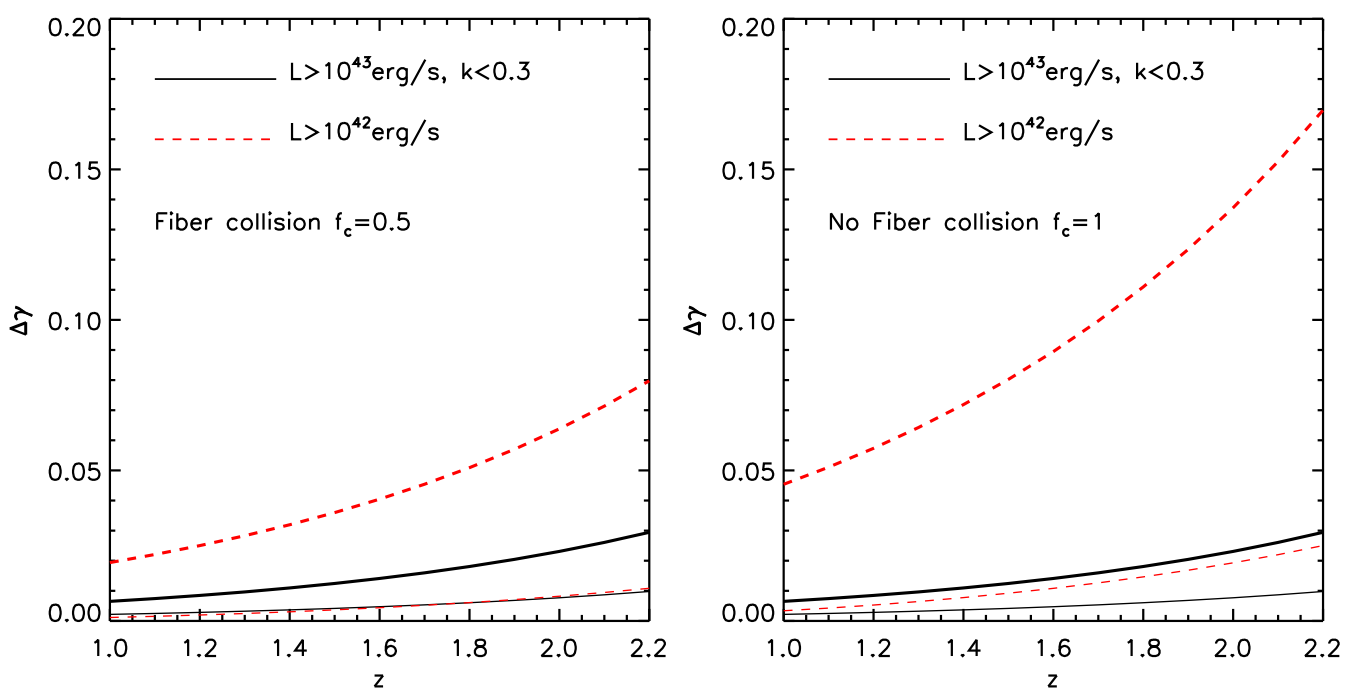

Figure 15. $\Delta \gamma$ as a function of the redshift for the $\mathrm{H} \alpha$ emitter sample $L>10^{43} \mathrm{erg} / \mathrm{s}$ (solid curve) and $L>10^{42} \mathrm{erg} / \mathrm{s}$ (dashed curve). The left panel is no fiber collision $f_{\text {col }}=1$, while the right panel is the case with the fiber collision $f_{\text {col }}=0.5$. In each panel, the thin curve is the case A estimation, but the thick curve is the case $\mathrm{B}$ estimation.

expressions

$$
\begin{aligned}
& F_{i j}^{\theta \theta}=\frac{1}{8 \pi^{2}} \int_{k_{\min }}^{k_{\max }} d k k^{2} \int_{-1}^{+1} d \mu \frac{\partial P(k, \mu)}{\partial \theta_{i}} \frac{\partial P(k, \mu)}{\partial \theta_{j}} \frac{V}{[P(k, \mu)+1 / \bar{n}]^{2}}, \\
& F_{i j}^{\theta \psi} \delta \psi_{j}=\frac{1}{8 \pi^{2}} \int_{k_{\min }}^{k_{\max }} d k k^{2} \int_{-1}^{+1} d \mu \frac{\partial P(k, \mu)}{\partial \theta_{i}} P^{1 h}(k, \mu) \frac{V}{[P(k, \mu)+1 / \bar{n}]^{2}},
\end{aligned}
$$

where $V$ is a survey volume, and we set $k_{\min }=0.01 h \mathrm{Mpc}^{-1}$ and $k_{\max }=0.3 h \mathrm{Mpc}^{-1}$. In case $B$, we use [45]

$$
\begin{aligned}
& F_{i j}^{\theta \theta}=\sum_{\ell=0,2, \cdots}^{\ell_{\max }=6} \frac{1}{4 \pi^{2}} \int_{k_{\min }}^{k_{\max }} d k k^{2} \frac{\partial P_{\ell}(k)}{\partial \theta_{i}} \frac{\partial P_{\ell}(k)}{\partial \theta_{j}} \kappa(k), \\
& F_{k j}^{\theta \psi} \delta \psi_{j}=\sum_{\ell=0,2, \cdots}^{\ell_{\max }=6} \frac{1}{4 \pi^{2}} \int_{k_{\min }}^{k_{\max }} d k k^{2} \frac{\partial P_{\ell}(k)}{\partial \theta_{i}} P_{\ell}^{1 h}(k) \kappa(k)
\end{aligned}
$$

with

$$
\kappa(k)=\frac{1}{2 \ell+1} \frac{V}{\left[P_{0}(k)+1 / \bar{n}\right]^{2}} .
$$

In the above expressions, we consider the power spectrum that is the combination of the one-halo term (3.2) and the two-halo term (3.11), with the growth rate $f=\Omega_{m}(z)^{\gamma}$,

$$
\begin{aligned}
& b(M)=\left(b_{0}+b_{1} k\right) b_{\text {halo }}(M), \\
& \tilde{p}_{c s}(k, \mu ; M)=e^{-\alpha^{2} \sigma_{v, o f f}^{2}(M) k^{2} \mu^{2} / 2 a^{2} H^{2}},
\end{aligned}
$$


where $\gamma, b_{0}, b_{1}, \alpha$ are parameters, and $b_{\text {halo }}(M)$ is the halo bias, fixed as [46]

$$
b_{\text {halo }}(M)=1-\frac{\nu^{a}}{\nu^{a}+\delta_{c}^{a}}+0.183 \nu^{b}+0.265 \nu^{c},
$$

with $\nu=\delta_{c} / \sigma(M, z), \delta_{c}=1.686, a=0.132, b=1.5$ and $c=2.4$. We adopt the 4 parameters, $\gamma, b_{0}, b_{1}, \alpha$ for the Fisher matrix analysis, where the target parameter is $\gamma=0.55, b_{0}=1$, $b_{1}=0.2, \alpha=1$. The background cosmology is fixed to be the $\Lambda$ CDM model with $\Omega_{m}=0.3$, $\Omega_{b}=0.044$, and $\sigma_{8}=0.8$.

In the present paper, we focus on the systematic bias in $\gamma$, which is considered to be useful for testing gravity. Figure 15 shows the systematic bias $\Delta \gamma$ as a function of the redshift. In each panel, the solid curve (dashed curve) adopts the HOD with $L>10^{43} \mathrm{erg} / \mathrm{s}\left(L>10^{42}\right.$ $\mathrm{erg} / \mathrm{s}$ ), and the thin (thick) curve is the case $A$ (case $B$ ) for the estimation of the Fisher matrix, respectively. The left panel assumes no Fiber collision, while the right panel take the fiber collision into account by assuming $f_{\text {col }}=0.5$.

Figure 15 means that the fiber collision reduces the systematic bias because the satellite fraction, which causes the systematic error, is reduced. Furthermore, brighter $\mathrm{H} \alpha$ emitters do not generally contain satellite, which also reduces the systematic bias. The mean number density is $\bar{n} \simeq(2 \sim 3) \times 10^{-4}(h / \mathrm{Mpc})^{3}$ for the $\mathrm{H} \alpha$ emitter with $L>10^{43} \mathrm{erg} / \mathrm{s}$ (solid curve), while $\bar{n} \simeq(2 \sim 3) \times 10^{-3}(h / \mathrm{Mpc})^{3}$ for $\mathrm{H} \alpha$ emitter with $L>10^{42} \mathrm{erg} / \mathrm{s}$ (dashed curve). The number density of galaxies of a optimized redshift survey would be $\bar{n} \simeq(2 \sim$ $3) \times 10^{-4}(h / \mathrm{Mpc})^{3}$. In this case, the sample with $L>10^{43} \mathrm{erg} / \mathrm{s}$ (solid curve) will be a realistic sample, whose systematic bias in $\gamma$ is not large. It might be worthy to note that an analysis with the multipole power spectrum (case $B$ : thick curve) makes a larger systematic bias compared with an analysis with the full anisotropic power spectrum (case A: thin curve).

In general, the amplitude of the one-halo term becomes smaller at higher redshift because the halo mass becomes smaller. However, the power spectrum is less sensitive to the cosmological parameter at higher redshift, which reduces the Fisher matrix elements at higher redshift. This is one of the reason why the systematic bias becomes larger at higher redshift. In the present paper, we omitted the random velocity dispersion between halos. For the $\mathrm{H} \alpha$ emitters, however, the halo random velocity could be large. This effect will be included in the two halo term, but not in the one halo term. Then, this might not be included as an uncertainty of the one halo term, but is related with the modeling of the two halo term. A more precise theoretical model for the $\mathrm{H} \alpha$ emitters will be necessary including the HOD model and the fiber collision, depending on observational strategy. Our results here are obtained by extensively using the HOD model, which was originally obtained at $z>2$.

\section{Summary and Conclusions}

In the present paper, we have investigated the influence of the satellite galaxies on the redshift-space distortions. We have found the following points, for the first time. First, the satellite galaxies significantly contribute to the higher-order multipole power spectrum though the fraction is small. Second, the contribution of the satellite galaxies to the higherorder multipole power spectrum is explained by a simple halo model, and the one halo term makes the dominant contribution. We have also demonstrated that the contribution from satellite galaxies depends on the HOD of galaxy samples and the effect of the fiber collision. These findings are based on the SDSS LRG sample, but generally means that an uncertainty of the HOD might give rise to a systematic error in measuring redshift-space distortion 
when satellite galaxies are contaminated. We have also demonstrated that the small-scale information of higher multipole spectra $P_{4}(k)$ and $P_{6}(k)$ at large wavenumbers help calibrate the satellite FoG effect and improve the measurement of growth rate dramatically.

For the $\mathrm{H} \alpha$ emitters, which are the target galaxies of the PFS redshift survey and the Euclid redshift survey, we have shown that the satellite's contribution to the redshift-space distortion is much smaller than the case of LRGs, because the host halo mass is small. The results are based on the HOD of the $\mathrm{H} \alpha$ emitters at the redshift $z>2$, it would be interesting to investigate how the results change depending on the redshift especially in the lower redshift regions. Combination with weak lensing survey might help to resolve the uncertainty in HOD [47-49]. A simple Fisher matrix analysis shows that the systematic error from the HOD uncertainty in the parameter $\gamma$ is not large for $\mathrm{H} \alpha$ emitters with $L>10^{43}$ $\mathrm{erg} / \mathrm{s}$. But this conclusion is based on the simple model with the HOD model, which was originally obtained at $z>2$. Then further check will be necessary, including a modeling of the peculiar velocity of halos.

The one-halo term makes the significant contribution to the higher multipole power spectrum of the LRG sample. It is expected that the same situation happens in the CMASS sample of the BOSS survey. The one-halo term reflects the HOD as well as the random velocities of satellite galaxies in a halo. This fact might provide us with an additional cosmological information on the scales of cluster of galaxies. For example, in a class of modified gravity model, the effective gravitational constant in a halo could be larger than that of the solar system. This enhances the velocity of satellite galaxies, which might be detected a signature of modified gravity theories (c.f. [50]). Such a signature might be constrained from the observation of higher multipole power spectrum. But we have also demonstrated that such a gravity-test requires the precise information of the velocity probability distribution function of satellite galaxies as well as the HOD, plus the fiber collision effect. This subject is also left as a future problem.

\section{Acknowledgments}

We thank M. Takada and S. Masaki for useful discussions at the early stage of this work. We also thank A. Oka, S. Saito, T. Nishimichi, A. Taruya, T. Matsubara, T. Okumura, T. Kanemaru, and A. Terukina for useful communications related to the topic of the present paper. We acknowledge anonymous referree for useful and constructive comments. The research by K.Y. and C.H. is supported in part by Grant-in-Aid for Scientific researcher of Japanese Ministry of Education, Culture, Sports, Science and Technology (No. 21540270 and No. 21244033 for K.Y. and No. 24740160 for C.H.). K.Y. is also supported by exchange visitor program between JSPS and DFG.

\section{A Derivation of power spectrum}

In this appendix, we derive a general expression of the multipole power spectrum in the halo model, which gives the grounds to adopt the expressions in section 3. Following the halo model approach, the correlation function is written as the sum of the 1-halo term and the 2-halo term. The power spectrum is the Fourier transform of the correlation function, then the power spectrum is also written as the combination of the 1-halo term and the 2-halo term. We start with the real-space power spectrum in a halo model presented in reference [51],

$$
P^{R}(k)=P^{R 1 h}(k)+P^{R 2 h}(k),
$$


where we defined

$$
\begin{aligned}
& P^{R 1 h}(k)=\frac{1}{\bar{n}^{2}} \int d M \frac{d n(M)}{d M}\left\langle N_{c e n}\right\rangle\left[2\left\langle N_{\text {sat }}\right\rangle \tilde{u}_{\mathrm{NFW}}(k ; M)+\left\langle N_{\text {sat }}\left(N_{\text {sat }}-1\right)\right\rangle \tilde{u}_{\mathrm{NFW}}(k ; M)^{2}\right], \\
& P^{R 2 h}(k)=\frac{1}{\bar{n}^{2}}\left[\int d M \frac{d n(M)}{d M}\left\langle N_{c e n}\right\rangle\left(1+\left\langle N_{\text {sat }}\right\rangle \tilde{u}_{\mathrm{NFW}}(k ; M)\right) b(M)\right]^{2} P_{m}(k),
\end{aligned}
$$

and $\tilde{u}_{\mathrm{NFW}}(k ; M)$ is the Fourier transform of the density profile of galaxy distribution. We assume that the galaxy density profile is the same as the dark matter density profile. For the NFW density profile, we have $[27,32]$

$$
\begin{aligned}
\tilde{u}_{\mathrm{NFW}}(k ; M)= & \frac{\int_{r \leq r_{v i r}} d^{3} x \rho(x \mid M) e^{-i \mathbf{k} \cdot \mathbf{x}}}{\int_{r \leq r_{v i r}} d^{3} x \rho(x \mid M)} \\
= & \frac{4 \pi \rho_{s} r_{s}^{3}}{M}\left\{\sin \left(k r_{s}\right)\left[S i\left([1+c] k r_{s}\right)-S i\left(k r_{s}\right)\right]\right. \\
& \left.-\frac{\sin c k r_{s}}{(1+c) k r_{s}}+\cos \left(k r_{s}\right)\left[C i\left([1+c] k r_{s}\right)-C i\left(k r_{s}\right)\right]\right\},
\end{aligned}
$$

where

$$
C_{i}(x)=-\int_{x}^{\infty} \frac{\cos t}{t} d t, \quad S_{i}(x)=\int_{0}^{x} \frac{\sin t}{t} d t .
$$

The redshift-space power spectrum of the halo model may be evaluated as follows. Tinker investigated the formulation for the redshift-space correlation function in a halo model [52], in which the redshift-space correlation function is obtained by $[36,53]$

$$
\xi\left(s_{\perp}, s_{\|}\right)=\int \xi^{R}(r) \mathcal{P}\left(v_{z}\right) d v_{z},
$$

where $\xi^{R}(r)$ is the real-space correlation function, $s_{\perp}$ is the projected separation, $s_{\|}$is the line of sight separation, $r^{2}=s_{\perp}^{2}+z^{2}$ and $v_{z}=H\left(s_{\|}-z\right), \mathcal{P}\left(v_{z}\right)$ is the probability distribution function of the galaxy pairwise velocity, and $H$ is the Hubble parameter. $\mathcal{P}\left(v_{z}\right)$ maps the pairs at separation in the line-of-sight direction $z$ to $s_{\|}$with the probability $\mathcal{P}\left(v_{z}\right)[36,53]$. This gives the prescription to include the random velocity of galaxies in a halo in redshift-space power spectrum. Then, we may write the redshift-space power spectrum in the form

$$
P(k, \mu)=P^{1 h}(k, \mu)+P^{2 h}(k, \mu),
$$

where

$$
\begin{aligned}
P^{1 h}(k, \mu)=\frac{1}{\bar{n}^{2}} \int d M \frac{d n(M)}{d M} & \left\langle N_{c e n}\right\rangle\left[2\left\langle N_{\text {sat }}\right\rangle \tilde{p}_{c s}(k, \mu ; M)\right. \\
\times & \left.+\left\langle N_{\text {sat }}\left(N_{\text {sat }}-1\right)\right\rangle \tilde{p}_{s s}(k, \mu ; M)\right],
\end{aligned}
$$

and

$$
\begin{aligned}
P^{2 h}(k, \mu)=[ & \frac{1}{\bar{n}} \int d M \frac{d n(M)}{d M}\left\langle N_{c e n}\right\rangle \\
& \left.\times\left(1+\left\langle N_{s a t}\right\rangle \tilde{p}_{c s}(k, \mu ; M)\right)\left(b(M)+f \mu^{2}\right)\right]^{2} P_{m}(k),
\end{aligned}
$$


where we defined

$$
\tilde{p}_{c s}(k, \mu ; M)=\tilde{u}_{\mathrm{NFW}}(k ; M) e^{-\sigma_{v}^{2} k^{2} \mu^{2} / 2 a^{2} H^{2}},
$$

when the pair wise velocity between the central galaxy and the satellite galaxy obeys the Gaussian probability distribution function $\mathcal{P}\left(v_{z}\right)=\left(\sqrt{2 \pi} \sigma_{v}\right)^{-1} e^{-v_{z}^{2} / 2 \sigma_{v}^{2}}$. Here we assume that the random velocity of the central galaxies can be neglected, then we may write $\tilde{p}_{s s}(k, \mu ; M)=$ $\tilde{p}_{c s}^{2}(k, \mu ; M)$ for the satellite-satellite galaxy pair. In the case of the exponential velocity distribution function, $\mathcal{P}\left(v_{z}\right)=\left(\sqrt{2} \sigma_{v}\right)^{-1} e^{-\sqrt{2}\left|v_{z}\right| / \sigma}$, we have

$$
\begin{aligned}
& \tilde{p}_{c s}(k, \mu ; M)=\frac{\tilde{u}_{\mathrm{NFW}}(k ; M)}{1+\sigma_{v}^{2} k^{2} \mu^{2} / 2 a^{2} H^{2}}=\tilde{u}_{\mathrm{NFW}}(k ; M) \mathcal{D}\left(\frac{\sigma_{v} k \mu}{a H}\right), \\
& \tilde{p}_{s s}(k, \mu ; M)=\frac{\tilde{u}_{\mathrm{NFW}}(k ; M)^{2}}{1+\sigma_{v}^{2} k^{2} \mu^{2} / a^{2} H^{2}}=\tilde{u}_{\mathrm{NFW}}(k ; M)^{2} \mathcal{D}\left(\frac{\sqrt{2} \sigma_{v} k \mu}{a H}\right) .
\end{aligned}
$$

As is shown in section 3, the one-halo term dominates the higher multipole power spectrum of the All LRG sample. It is useful to present the analytic formula, as is given by equation (3.12) with (3.14)-(3.17) for the case of the Gaussian velocity distribution function. In the case of the exponential velocity distribution function, (3.14)-(3.17) are replaced with

$$
\begin{aligned}
Q_{0}(q) & =\frac{\arctan q}{q} \\
Q_{2}(q) & =\frac{3 q-\left(3+q^{2}\right) \arctan q}{2 q^{3}} \\
Q_{4}(q) & =\frac{-105 q-55 q^{3}+\left(105+90 q^{2}+9 q^{4}\right) \arctan q}{24 q^{5}}, \\
Q_{6}(q) & =\frac{1155 q+1190 q^{3}+231 q^{5}-\left(1155+1575 q^{2}+525 q^{4}+25 q^{6}\right) \arctan q}{80 q^{7}} .
\end{aligned}
$$

\section{References}

[1] W. J. Percival, B. A. Reid, D. J. Eisenstein, N. A. Bahcall, T. Budavari, J. A. Frieman, M. Fukugita, J. E. Gunn, Z. Ivezic, G. R. Knapp, et al., Mon. Not. Roy. Astron. Soc., 401 (2010) 2148

[2] A. Cabre and E. Gaztanaga, Mon. Not. Roy. Astron. Soc., 396 (2009) 1119

[3] B. A. Reid, W. J. Percival, D. J. Eisenstein, L. Verde, D. N. Spergel, R. A. Skibba, N. A. Bahcall, T. Budavari, J. A. Frie- man, M. Fukugita, et al., Mon. Not. Roy. Astron. Soc., 404 (2010) 60

[4] S. Saito, M. Takada, and A. Taruya, Phys. Rev. D 83 (2011) 043529

[5] K. Yamamoto, T. Sato, and G. Huetsi, Progress of Theoretical Physics, 120 (2008) 609

[6] K. Yamamoto, G. Nakamura, G. Huetsi, T. Narikawa, and T. Sato, Phys. Rev. D 81 (2010) 103517

[7] L. Guzzo et al., Nature (London) 451 (2008) 541

[8] B. A. Reid and D. N. Spergel, Astrophys. J. 698 (2009) 143

[9] Z. Zheng, I. Zehavi, D. J. Eisenstein, D. H. Weinberg, and Y. P.Jing, Astrophys. J. 707 (2009) 554 
[10] B. A. Reid, D. N. Spergel, and P. Bode, Astrophys. J. 702 (2009) 249

[11] C. Hikage, M. Takada, and D. N. Spergel, Mon. Not. Roy. Astron. Soc. 419 (2012) 3457

[12] C. Hikage, R. Mandelbaum, M. Takada, and D. N. Spergel, ArXiv e-prints (2012), 1211.1009

[13] J. C. Jackson, Mon. Not. Roy. Astron. Soc. 156 (1972) 156

[14] S. Masaki, C. Hikage, M. Takada, D. N. Spergel, and N. Sugiyama, Mon. Not. Roy. Astron. Soc. accepted, ArXiv e-prints (2012), 1211.7077

[15] J. A. Peacock et al., Nature (London) 410 (2001) 169

[16] I. Zehavi, M. R. Blanton, J. A. Frieman, D. H. Weinberg, H. J. Mo, M. A. Strauss, S. F. Anderson, J. Annis, N. A. Bahcall, M. Bernardi, et al., Astrophys. J. 571 (2002) 172

[17] A. Cabre and E. Gaztanaga, Mon. Not. Roy. Astron. Soc. 393 (2009) 1183

[18] T. Okumura, T. Matsubara, D. J. Eisenstein, I. Kayo, C. Hik- age, A. S. Szalay, and D. P. Schneider, Astrophys. J. 676 (2008) 889

[19] U. Seljak and P. McDonald, JCAP 11 (2011) 039

[20] T. Okumura, U. Seljak, and V. Desjacques, JCAP 11(2012) 014

[21] T. Sato, G. Huetsi, and K. Yamamoto, Progress of Theoretical Physics 125 (2011) 187

[22] E. A. Kazin, M. R. Blanton, R. Scoccimarro, C. K. McBride, A. A. Berlind, N. A. Bahcall, J. Brinkmann, P. Czarapata, J. A. Frieman, S. M. Kent, et al., Astrophys. J. 710 (2010) 1444

[23] K. Yamamoto, M. Nakamichi, A. Kamino, B. A. Bassett, and H. Nishioka, Publ. of Astron. Soc. of Japan $\mathbf{5 8}$ (2006) 93

[24] E. V. Linder, Phys. Rev. D 72 (2005) 043529

[25] U. Seljak, Mon. Not. Roy. Astron. Soc. 318 (2000) 203

[26] M. White, Mon. Not. Roy. Astron. Soc. 321 (2001) 1

[27] A. Cooray and R. Sheth, Phys. Rep. 372 (2002) 1

[28] R. K. Sheth and G. Tormen, Mon. Not. Roy. Astron. Soc. 308 (1999) 119

[29] Z. Zheng et al., Astrophys. J. 633 (2005) 791

[30] A. V. Kravtsov, A. A. Berlind, R. H. Wechsler, A. A. Klypin, S. Gottlo ber, B. Allgood, and J. R. Primack, Astrophys. J. 609 (2004) 35

[31] J. F. Navarro, C. S. Frenk, and S. D. M. White, Astrophys. J. 490 (1997) 493

[32] R. Scoccimarro, R. K. Sheth, L. Hui, and B. Jain, Astrophys. J. 546 (2001) 20

[33] N. Kaiser, Mon. Not. Roy. Astron. Soc. 227 (1987) 1

[34] H. J. Mo, Y. P. Jing, and S. D. M. White, Mon. Not. Roy. Astron. Soc. 284 (1997) 189

[35] R. A. Skibba, F. C. van den Bosch, X. Yang, S. More, H. Mo, and F. Fontanot, Mon. Not. Roy. Astron. Soc. 410 (2011) 417

[36] R. Scoccimarro, Phys. Rev. D 70 (2004) 083007

[37] T. Matsubara, Phys. Rev. D 77 (2008) 063530

[38] A. Taruya, T. Nishimichi, and S. Saito, Phys. Rev. D 82 (2010) 063522

[39] T. Nishimichi and A. Taruya, Phys. Rev. D 84 (2011) 043526

[40] R. Ellis, M. Takada, H. Aihara, N. Arimoto, K. Bundy, M. Chiba, J. Cohen, O. Dore, J. E. Greene, J. Gunn, et al., arXiv e-prints (2012), 1206.0737

[41] R. Laureijs, J. Amiaux, S. Arduini, J. . Augue'res, J. Brinch- mann, R. Cole, M. Cropper, C. Dabin, L. Duvet, A. Ealet, et al., ArXiv e-prints (2011), 1110.3193 
[42] J. E. Geach, D. Sobral, R. C. Hickox, D. A. Wake, I. Smail, P. N. Best, C. M. Baugh, and J. P. Stott, Mon. Not. Roy. Astron. Soc. 426 (2012) 679

[43] A. N. Taylor, T. D. Kitching, D. J. Bacon, A. F. Heavens, Mon. Not. Roy. Astron. Soc. 374 (2007) 1377

[44] L. Knox, R. Scoccimarro, and S. Dodelson, Phys. Rev. Lett. 81, (1998) 2004

[45] K. Yamamoto, B. A. Bassett, H. Nishioka, Phys. Rev. Lett. 94, (2005) 051301

[46] J. Tinker, et al., Astrophys. J. 724, (2010) 878

[47] F. C. van den Bosch, S. More, M. Cacciato, H. Mo, and X. Yang, Mon. Not. Roy. Astron. Soc. 430 (2013) 725

[48] S. More, F. C. van den Bosch, M. Cacciato, A. More, H. Mo, and X. Yang, Mon. Not. Roy. Astron. Soc. 430 (2013) 747

[49] M. Cacciato, F. C. van den Bosch, S. More, H. Mo, and X. Yang, Mon. Not. Roy. Astron. Soc. 430 (2013) 767

[50] T. Nishimichi, Phys. Rev. Lett. 109, (2012) 051301

[51] R. A. Skibba and R. K. Sheth, Mon. Not. Roy. Astron. Soc. 392, (2009) 1080

[52] J. L. Tinker, Mon. Not. Roy. Astron. Soc. 374, (2007) 477

[53] P. J. E. Peebles, Astron. J. 84, (1979) 730 